\title{
ILLUMINATING A DARK LENS: A TYPE Ia SUPERNOVA MAGNIFIED BY THE FRONTIER FIELDS GALAXY CLUSTER ABELL 2744
}

\author{
Steven A. Rodney ${ }^{1,2,34}$, Brandon Patel ${ }^{3}$, Daniel Scolnic ${ }^{4}$, Ryan J. Foley ${ }^{5,6}$, Alberto Molino ${ }^{7,8}$, Gabriel Brammer ${ }^{9}$, \\ Mathilde Jauzac ${ }^{10,11}$, Maruša Bradač ${ }^{12}$, Tom Broadhurst ${ }^{13,14}$, Dan Coe ${ }^{9}$, Jose M. Diego ${ }^{15}$, Or Graur ${ }^{16,17}$, Jens Huorth ${ }^{18}$, \\ Austin Hoag $^{12}$, Saurabh W. Jha ${ }^{3}$, Traci L. Johnson ${ }^{19}$, Patrick Kelly ${ }^{20}$, Daniel Lam ${ }^{21}$, Curtis McCully ${ }^{22,23}$, \\ Elinor Medezinski $^{2,24}$, Massimo Meneghetti ${ }^{25,26,27}$, Julian Merten ${ }^{28}$, Johan Richard ${ }^{29}$, Adam Riess ${ }^{2,9}$, Keren Sharon $^{19}$, \\ Louis-Gregory Strolger ${ }^{9,30}$, Tommaso Treu ${ }^{31,35}$, Xin Wang ${ }^{23}$, Liliya L. R. Williams ${ }^{32}$, AND Adi Zitrin ${ }^{33,34}$ \\ ${ }^{1}$ Department of Physics and Astronomy, University of South Carolina, 712 Main St., Columbia, SC 29208, USA; srodney@sc.edu \\ ${ }^{2}$ Department of Physics and Astronomy, The Johns Hopkins University, 3400 N. Charles St., Baltimore, MD 21218, USA \\ ${ }_{3}^{3}$ Department of Physics and Astronomy, Rutgers, The State University of New Jersey, Piscataway, NJ 08854, USA \\ ${ }_{5}^{4}$ Kavli Institute for Cosmological Physics, University of Chicago, 5640 South Ellis Avenue Chicago, IL 60637, USA \\ 5 Department of Physics, University of Illinois at Urbana-Champaign, 1110 W. Green Street, Urbana, IL 61801, USA \\ ${ }^{6}$ Astronomy Department, University of Illinois at Urbana-Champaign, 1002 W. Green Street, Urbana, IL 61801, USA \\ ${ }^{7}$ Instituto de Astrofísica de Andalucía (CSIC), E-18080 Granada, Spain \\ ${ }^{8}$ Instituto de Astronomia, Geofísica e Ciências Atmosféricas, Universidade de São Paulo, Cidade Universitária, 05508-090, São Paulo, Brazil \\ ${ }^{9}$ Space Telescope Science Institute, 3700 San Martin Dr., Baltimore, MD 21218, USA \\ ${ }^{10}$ Institute for Computational Cosmology, Durham University, South Road, Durham DH1 3LE, UK \\ ${ }^{11}$ Astrophysics and Cosmology Research Unit, School of Mathematical Sciences, University of KwaZulu-Natal, Durban 4041, South Africa \\ ${ }^{12}$ University of California Davis, 1 Shields Avenue, Davis, CA 95616, USA \\ ${ }^{13}$ Fisika Teorikoa, Zientzia eta Teknologia Fakultatea, Euskal Herriko Unibertsitatea UPV/EHU, Spain \\ ${ }^{14}$ IKERBASQUE, Basque Foundation for Science, Alameda Urquijo 36-5, E-48008 Bilbao, Spain \\ ${ }^{15}$ IFCA, Instituto de Física de Cantabria (UC-CSIC), Av. de Los Castros s/n, E-39005 Santander, Spain \\ ${ }^{16}$ Center for Cosmology and Particle Physics, New York University, New York, NY 10003, USA \\ ${ }_{17}$ Department of Astrophysics, American Museum of Natural History, Central Park West and 79th Street, New York, NY 10024, USA \\ ${ }^{18}$ Dark Cosmology Centre, Niels Bohr Institute, University of Copenhagen, Juliane Maries Vej 30, DK-2100 Copenhagen, Denmark \\ ${ }^{19}$ Department of Astronomy, University of Michigan, 1085 S. University Avenue, Ann Arbor, MI 48109, USA \\ ${ }^{20}$ Department of Astronomy, University of California, Berkeley, CA 94720-3411, USA \\ ${ }^{21}$ Department of Physics, The University of Hong Kong, Pokfulam Road, Hong Kong, China \\ ${ }^{22}$ Las Cumbres Observatory Global Telescope Network, 6740 Cortona Dr., Suite 102, Goleta, CA 93117, USA \\ ${ }^{23}$ Department of Physics, University of California, Santa Barbara, CA 93106-9530, USA \\ ${ }^{24}$ The Hebrew University, The Edmond J. Safra Campus-Givat Ram, Jerusalem 9190401, Israel \\ 25 INAF, Osservatorio Astronomico di Bologna, Via Ranzani 1, I-40127 Bologna, Italy
${ }^{26}$ Jet Propulsion Laboratory, California Institute of Technology, 4800 Oak Grove Drive, Pasadena, CA 91109, USA \\ ${ }^{27}$ INFN, Sezione di Bologna, Viale Berti Pichat 6/2, I-40127 Bologna, Italy \\ ${ }^{28}$ Department of Physics, University of Oxford, Keble Road, Oxford OX1 3RH, UK \\ ${ }^{29}$ CRAL, Observatoire de Lyon, Université Lyon 1, 9 Avenue Ch. André, F-69561 Saint Genis Laval Cedex, France \\ ${ }^{30}$ Department of Physics, Western Kentucky University, Bowling Green, KY 42101, USA \\ ${ }^{31}$ Department of Physics and Astronomy, University of California, Los Angeles, CA 90095, USA \\ ${ }^{32}$ School of Physics and Astronomy, University of Minnesota, 116 Church Street SE, Minneapolis, MN 55455, USA \\ ${ }^{33}$ Cahill Center for Astronomy and Astrophysics, California Institute of Technology, MC 249-17, Pasadena, CA 91125, USA \\ Received 2015 May 22; accepted 2015 August 14; published 2015 September 22
}

\begin{abstract}
SN HFF14Tom is a Type Ia SN discovered at $z=1.3457 \pm 0.0001$ behind the galaxy cluster Abell 2744 $(z=0.308)$. In a cosmology-independent analysis, we find that HFF14Tom is $0.77 \pm 0.15$ mag brighter than unlensed Type Ia SNe at similar redshift, implying a lensing magnification of $\mu_{\mathrm{obs}}=2.03 \pm 0.29$. This observed magnification provides a rare opportunity for a direct empirical test of galaxy cluster lens models. Here we test 17 lens models, 13 of which were generated before the SN magnification was known, qualifying as pure "blind tests." The models are collectively fairly accurate: 8 of the models deliver median magnifications that are consistent with the measured $\mu$ to within $1 \sigma$. However, there is a subtle systematic bias: the significant disagreements all involve models overpredicting the magnification. We evaluate possible causes for this mild bias, and find no single physical or methodological explanation to account for it. We do find that model accuracy can be improved to some extent with stringent quality cuts on multiply imaged systems, such as requiring that a large fraction have spectroscopic redshifts. In addition to testing model accuracies as we have done here, Type Ia SN magnifications could also be used as inputs for future lens models of Abell 2744 and other clusters, providing valuable constraints in regions where traditional strong- and weak-lensing information is unavailable.
\end{abstract}

Key words: galaxies: clusters: general - galaxies: clusters: individual (Abell 2744) - gravitational lensing: strong gravitational lensing: weak - supernovae: general - supernovae: individual (HFF14Tom)

\footnotetext{
34 Hubble Fellow.

35 Packard Fellow.
}

\section{INTRODUCTION}

Galaxy clusters can be used as cosmic telescopes to magnify distant background objects through gravitational lensing, which can substantially increase the reach of deep imaging surveys. 
The lensing magnification enables the study of objects that would otherwise be unobservable because they are either intrinsically faint (e.g., Schenker et al. 2012; Alavi et al. 2014) or extremely distant (e.g., Franx et al. 1997; Ellis et al. 2001; $\mathrm{Hu}$ et al. 2002; Kneib et al. 2004; Richard et al. 2006, 2008; Bouwens et al. 2009; Maizy et al. 2010; Zheng et al. 2012; Coe et al. 2013; Bouwens et al. 2014; Zitrin et al. 2014). Background galaxies are also spatially magnified, allowing for studies of the internal structure of galaxies in the early universe with resolutions of $\sim 100$ pc (e.g., Stark et al. 2008; Jones et al. 2010; Yuan et al. 2011; Wuyts et al. 2014; Livermore et al. 2015).

Gravitational lensing can also provide a powerful window onto the transient sky through an appropriately cadenced imaging survey. The flux magnification from strong-lensing clusters is especially valuable for the study of $z>1.5$ supernovae (SNe) (e.g., Kovner \& Paczynski 1988; Kolatt \& Bartelmann 1998; Saini et al. 2000; Sullivan et al. 2000; Gunnarsson \& Goobar 2003; Goobar et al. 2009; Postman et al. 2012), which are still extremely difficult to characterize in unlensed fields (e.g., Riess et al. 2001, 2007; Rodney et al. 2012; Suzuki et al. 2012; Jones et al. 2013; Rubin et al. 2013).

In the case of lensed $\mathrm{SNe}$, we can also reverse the experimental setup: instead of using strong-lensing clusters to study distant $\mathrm{SNe}$, we can use the $\mathrm{SNe}$ as tools for examining the lenses (Riehm et al. 2011). The most valuable transients for testing and improving cluster lens models would be strongly lensed SNe that are resolved into multiple images (Holz 2001; Oguri \& Kawano 2003). Transients that are lensed into multiple images can also become cosmological tools, as the measurement of time delays between the images can provide cosmographic information to constrain the Hubble parameter (Refsdal 1964) and other cosmological parameters (Linder 2011). We have recently observed the first example of a multiply imaged SN (Kelly et al. 2015). We expect to detect the reappearance of this object (called "SN Refsdal") within the next year (Diego et al. 2015; Oguri 2015; Sharon \& Johnson 2015), delivering a precise test of lens model predictions. Although detections of such objects are currently very unlikely (Li et al. 2012), they will become much more common in the next decade (Coe \& Moustakas 2009; Dobke et al. 2009), and may be developed into an important new cosmological tool (Oguri 2010; Linder 2011).

In addition to time delays from multiply imaged $\mathrm{SNe}$, we can also put cluster mass models to the test with the much more common category of Type Ia SNe that are magnified but not multiply imaged. (Patel et al. 2014, hereafter P14) and Nordin et al. (2014) presented independent analyses of three lensed $\mathrm{SNe}$, of which at least two are securely classified as Type Ia $\mathrm{SNe}$ - all found in the Cluster Lensing and Supernova Survey with Hubble (CLASH, PI:Postman, Hubble Space Telescope (HST) Program ID 12068, Postman et al. 2012). Both groups demonstrated that these standard candles can be used to provide accurate and precise measurements of the true absolute magnification along a random sight line through the cluster. Although in these cases the $\mathrm{SNe}$ were used to test the cluster mass models, one could in principle incorporate the measured magnifications of Type Ia SNe into the cluster as additional model constraints. In that role, Type Ia SNe have the particular value that they can be found anywhere in the cluster field. Thus, they can deliver model constraints in regions of "middle distance" from the cluster core, where both strong- and weak- lensing constraints are unavailable. Moreover, given enough time, multiple background Type Ia $\mathrm{SNe}$ could be measured behind the same cluster, each providing a new magnification constraint.

One of the key values in observing standard candles behind gravitational lenses is in addressing the problem of the masssheet degeneracy (Falco et al. 1985; Schneider \& Seitz 1995). This degeneracy arises because one can introduce into a lens model an unassociated sheet of uniform mass in front of or behind the lens, without disturbing the primary observable quantities. For example, take a lens model with a given surface mass density $\kappa$, and then transform the surface mass density to $\kappa^{\prime}=(1-\lambda) \kappa+\lambda$ for any arbitrary value $\lambda$. Both the $\kappa$ and $\kappa^{\prime}$ models will produce exactly the same values for all positional and shear constraints from strong and weak lensing (Seitz \& Schneider 1997). When lensed background sources are available across a wide range of redshifts (as is the case for the Abell 2744 cluster discussed here), it should in principle be possible to break this degeneracy (Seitz \& Schneider 1997; Bradač et al. 2004). However, there are more complex versions of positional constraint degeneracies (Liesenborgs \& de Rijcke 2012; Schneider \& Sluse 2014). Such degeneracies do not extend to the absolute magnification of a background source's flux and size. In the case of the simple mass sheet degeneracy described above, the magnification scales as $\mu \propto(1-\lambda)^{-2}$ (see, e.g., Bartelmann 2010). Therefore, one can break these fundamental degeneracies with an absolute measurement of magnification from a standard candle (Holz 2001) or a standard ruler (Sonnenfeld et al. 2011).

In Section 2 we present the discovery and follow-up observations of SN HFF14Tom at $z=1.3457$, discovered behind the galaxy cluster Abell 2744. Section 3 examines the SN host galaxy. Sections 4 and 5 describe the spectroscopy and photometry of this SN, leading to a classification of the object as a normal Type Ia SN. In Section 6 we make a direct measurement of the magnification of this source due to gravitational lensing. Section 7 discusses the tension between our magnification measurement and the lens models. Our conclusions are summarized in Section 8, along with a discussion of future prospects.

\section{DISCOVERY, FOLLOW-UP, AND DATA PROCESSING}

SN HFF14Tom was discovered in HST observations with the Advanced Camera for Surveys (ACS) in the F606W and F814W bands ( $V$ and $I$ ), collected on UT 2014 May 15 as part of the Hubble Frontier Fields (HFF) survey (PI:J.Lotz, HSTPID:13495). ${ }^{36}$ The HFF program is a 3-year Director's discretionary initiative that is collecting 140 orbits of HST imaging (roughly $340 \mathrm{ks}$ ) on six massive galaxy clusters, plus six accompanying parallel fields. Each field is observed in three optical bands (ACS F435W, F606W, and F814W) and four infrared (IR) bands (WFC3-IR F105W, F125W, F140W, and F160W), although the optical and IR imaging campaigns are separated by $\sim 6$ months. Abell 2744 was the first cluster observed, with IR imaging spanning 2013 October-November, and optical imaging from 2014 May-July. A composite image of the HFF data showing the $\mathrm{SN}$ is presented in Figure 1, and the locations of the cluster center, the $\mathrm{SN}$, and the presumed host galaxy are given in Table 1. The SN detection was made in difference images constructed using template imaging of Abell

\footnotetext{
${ }^{36}$ http://www.stsci.edu/hst/campaigns/frontier-fields
} 


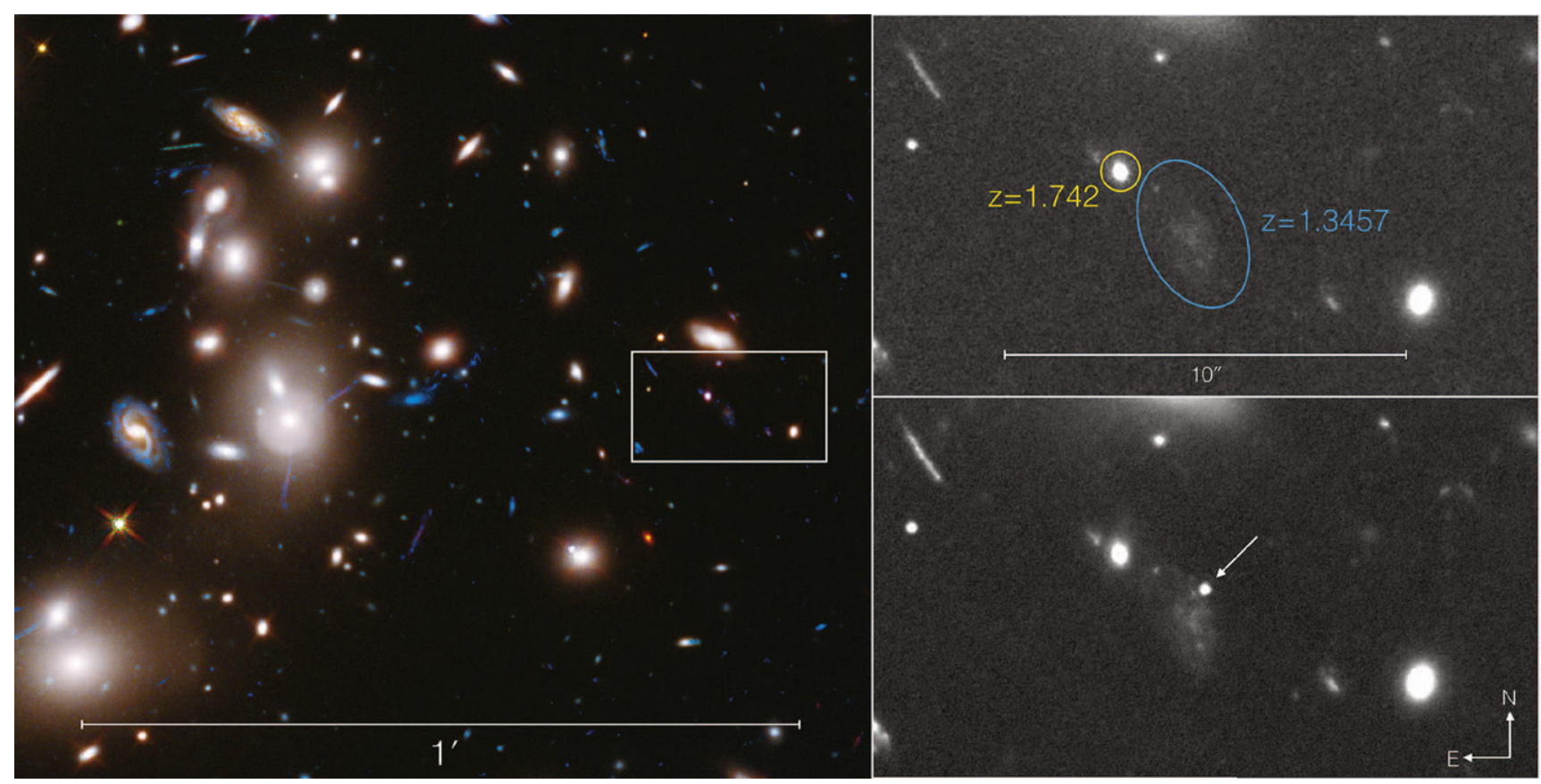

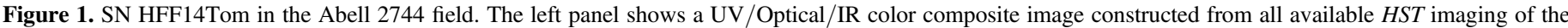

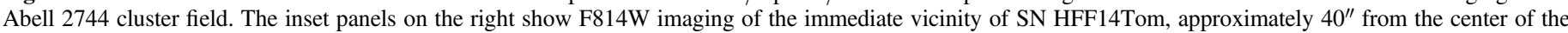

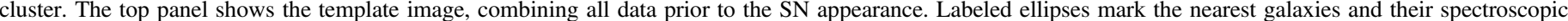

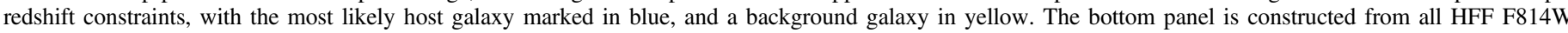

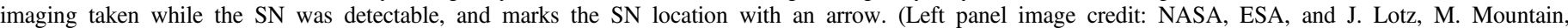
A. Koekemoer, and the HFF Team (STScI)).

Table 1

J2000 Coordinates of HFF14Tom, Host, and Cluster

\begin{tabular}{lcccc}
\hline \hline Object & $\begin{array}{c}\text { R.A. } \\
(\mathrm{h}: \mathrm{m}: \mathrm{s})\end{array}$ & $\begin{array}{c}\text { Decl. } \\
(\mathrm{d}: \mathrm{m}: \mathrm{s})\end{array}$ & $\begin{array}{l}\text { R.A. } \\
(\mathrm{deg})\end{array}$ & $\begin{array}{c}\text { Decl. } \\
(\mathrm{deg})\end{array}$ \\
\hline HFF14Tom & $00: 14: 17.87$ & $-30: 23: 59.7$ & 3.574458 & -30.399917 \\
Host galaxy $^{\text {Abell 2744 }}$ & $00: 14: 17.88$ & $-30: 24: 00.6$ & 3.574483 & -30.400175 \\
& $00: 14: 21.20$ & $-30: 23: 50.1$ & 3.588333 & -30.397250
\end{tabular}

\section{Note.}

a Coordinates of the HFF field center, approximately at the center of the cluster.

2744 from $H S T+$ ACS observations taken in 2009 (PI:Dupke, HST-PID:11689).

Upon discovery, HST target-of-opportunity observations were triggered from the FrontierSN program (PI:Rodney, HST-PID:13386), which aims to discover and follow transient sources in the HFF cluster and parallel fields. The FrontierSN observations provided WFC3-IR imaging as well as spectroscopy of the SN itself using the ACS G800L grism, supplementing the rapid-cadence optical imaging from $H S T+$ ACS already being provided by the HFF program. The last detections in the IR F105W and F140W bands came from the direct-imaging component of the Grism Lens-amplified Survey from Space (GLASS) program. Difference images for the IR follow-up data were generated using templates constructed from the HFF WFC3-IR imaging campaign, which concluded in 2013 November.

All of the imaging data were processed using the sndrizpipe pipeline, ${ }^{37}$ a custom data reduction package in Python that employs the DrizzlePac tools from the Space

\footnotetext{
${ }^{37}$ https://github.com/srodney/sndrizpipe v1.2 DOI:10.5281/zenodo.10731
}

Telescope Science Institute (STScI) (Fruchter et al. 2010). Photometry was collected using the PyPhot software package, ${ }^{38}$ a pure-Python implementation of the photometry algorithms from the IDL AstroLib package (Landsman 1993), which in turn are based on the DAOPHOT program (Stetson 1987). For the IR bands we used point-spread function fitting on the difference images, and in the ACS optical bands we collected photometry with a $0 . " 3$ aperture. Table 2 presents the list of observations, along with measured photometry from all available imaging data.

\section{HOST GALAXY}

The most probable host galaxy for SN HFF14Tom is a faint and diffuse galaxy immediately to the south-east of the SN location. With photometry of the host galaxy collected from the template images, we fit the spectral energy distribution (SED) using the BPZ code- a Bayesian photometric redshift estimator (Benítez 2000). From the BPZ analysis, we found the host to be most likely an actively star-forming galaxy at a redshift of $z=1.5 \pm 0.2$. This photo- $z$ was subsequently refined to a spectroscopic redshift of $z=1.3457 \pm 0.0001$, based on optical spectroscopy of the host (G. Mahler et al. 2015 , in preparation) that shows two significant $(>10 \sigma)$ emission lines at 8740.2 and $8746.7 \AA$, consistent with the [O II] $\lambda \lambda$ 3726-3729 A doublet.

The next nearest galaxy detected in HST imaging is 2.2 northeast of the SN position. It has a redshift of $z=1.742$ determined from a spectrum taken with the G141 grism of the HST WFC3-IR camera, collected as part of the GLASS (PI: Treu, PID:13459, Schmidt et al. 2014; Treu et al. 2015). As we

38 https://github.com/djones1040/PyPhot 
Table 2

HFF14Tom Observations and Photometry

\begin{tabular}{|c|c|c|c|c|c|c|c|c|c|}
\hline $\begin{array}{l}\text { Obs. Date } \\
\text { (MJD) }\end{array}$ & Camera & $\begin{array}{l}\text { Filter } \\
\text { or Grism }\end{array}$ & $\begin{array}{r}\text { Exp. Time } \\
\text { (s) }\end{array}$ & $\begin{array}{c}\text { Flux } \\
\left(\text { counts s }^{-1}\right)\end{array}$ & $\begin{array}{c}\text { Flux Err } \\
(\text { counts s } \\
-1\end{array}$ & $\overline{\mathrm{AB} \mathrm{Mag}}{ }^{\mathrm{a}}$ & Mag Err & AB Zero Point & $\begin{array}{c}\mathrm{ZP}^{\mathrm{b}} \\
(\mathrm{Vega}-\mathrm{AB})\end{array}$ \\
\hline 56820.06 & ACS & F435W & 5083 & -0.027 & 0.053 & 27.66 & $\ldots$ & 25.665 & -0.102 \\
\hline 56821.85 & $\mathrm{ACS}$ & F435W & 5083 & 0.105 & 0.053 & 28.11 & 0.55 & 25.665 & -0.102 \\
\hline 56823.77 & $\mathrm{ACS}$ & F435W & 5083 & 0.022 & 0.053 & 29.80 & 2.59 & 25.665 & -0.102 \\
\hline 56824.97 & $\mathrm{ACS}$ & F435W & 5083 & 0.021 & 0.053 & 29.85 & 2.72 & 25.665 & -0.102 \\
\hline 56828.68 & ACS & F435W & 5083 & -0.148 & 0.053 & 27.65 & $\ldots$ & 25.665 & -0.102 \\
\hline 56830.87 & $\mathrm{ACS}$ & F435W & 5083 & 0.100 & 0.054 & 28.16 & 0.58 & 25.665 & -0.102 \\
\hline 56832.86 & ACS & F435W & 5083 & -0.080 & 0.053 & 27.66 & $\ldots$ & 25.665 & -0.102 \\
\hline 56833.86 & $\mathrm{ACS}$ & F435W & 5083 & -0.002 & 0.053 & 27.67 & $\ldots$ & 25.665 & -0.102 \\
\hline 56839.50 & $\mathrm{ACS}$ & F435W & 5083 & -0.022 & 0.052 & 27.68 & $\ldots$ & 25.665 & -0.102 \\
\hline 56792.06 & ACS & F606W & 5046 & 0.363 & 0.083 & 27.59 & 0.25 & 26.493 & -0.086 \\
\hline 56792.98 & $\mathrm{ACS}$ & F606W & 3586 & 0.692 & 0.095 & 26.89 & 0.15 & 26.493 & -0.086 \\
\hline 56797.10 & $\mathrm{ACS}$ & F606W & 4977 & 0.968 & 0.087 & 26.53 & 0.10 & 26.493 & -0.086 \\
\hline 56800.08 & ACS & F606W & 4977 & 0.844 & 0.085 & 26.68 & 0.11 & 26.493 & -0.086 \\
\hline 56804.99 & $\mathrm{ACS}$ & F606W & 5046 & 0.977 & 0.086 & 26.52 & 0.10 & 26.493 & -0.086 \\
\hline 56792.99 & ACS & F814W & 3652 & 1.639 & 0.104 & 25.41 & 0.07 & 25.947 & -0.424 \\
\hline 56797.11 & ACS & F814W & 4904 & 3.376 & 0.141 & 24.63 & 0.05 & 25.947 & -0.424 \\
\hline 56798.95 & $\mathrm{ACS}$ & F814W & 5046 & 3.951 & 0.156 & 24.46 & 0.04 & 25.947 & -0.424 \\
\hline 56800.10 & ACS & F814W & 4904 & 3.854 & 0.155 & 24.48 & 0.04 & 25.947 & -0.424 \\
\hline 56801.89 & ACS & F814W & 10092 & 4.102 & 0.153 & 24.41 & 0.04 & 25.947 & -0.424 \\
\hline 56802.95 & ACS & F814W & 10092 & 4.325 & 0.160 & 24.36 & 0.04 & 25.947 & -0.424 \\
\hline 56803.93 & $\mathrm{ACS}$ & F814W & 15138 & 4.402 & 0.160 & 24.34 & 0.04 & 25.947 & -0.424 \\
\hline 56804.08 & ACS & F814W & 5046 & 4.658 & 0.178 & 24.28 & 0.04 & 25.947 & -0.424 \\
\hline 56812.08 & ACS & F814W & 637 & 4.705 & 0.258 & 24.27 & 0.06 & 25.947 & -0.424 \\
\hline 56815.93 & $\mathrm{ACS}$ & F814W & 446 & 4.026 & 0.285 & 24.43 & 0.08 & 25.947 & -0.424 \\
\hline 56820.07 & ACS & F814W & 5044 & 3.508 & 0.142 & 24.58 & 0.04 & 25.947 & -0.424 \\
\hline 56821.87 & ACS & F814W & 5044 & 3.541 & 0.144 & 24.57 & 0.04 & 25.947 & -0.424 \\
\hline 56823.79 & ACS & F814W & 5044 & 2.876 & 0.124 & 24.80 & 0.05 & 25.947 & -0.424 \\
\hline 56824.99 & ACS & F814W & 5044 & 3.060 & 0.129 & 24.73 & 0.05 & 25.947 & -0.424 \\
\hline 56828.70 & ACS & F814W & 5044 & 2.777 & 0.121 & 24.84 & 0.05 & 25.947 & -0.424 \\
\hline 56830.89 & ACS & F814W & 5044 & 2.395 & 0.111 & 25.00 & 0.05 & 25.947 & -0.424 \\
\hline 56832.88 & ACS & F814W & 5044 & 2.331 & 0.108 & 25.03 & 0.05 & 25.947 & -0.424 \\
\hline 56833.88 & ACS & F814W & 5044 & 2.389 & 0.111 & 25.00 & 0.05 & 25.947 & -0.424 \\
\hline 56839.52 & ACS & F814W & 5044 & 1.673 & 0.093 & 25.39 & 0.06 & 25.947 & -0.424 \\
\hline 56833.14 & WFC3-IR & F105W & 756 & 7.504 & 0.239 & 24.08 & 0.03 & 26.269 & -0.645 \\
\hline 56841.82 & WFC3-IR & F105W & 756 & 5.822 & 0.208 & 24.36 & 0.04 & 26.269 & -0.645 \\
\hline 56850.06 & WFC3-IR & F105W & 756 & 3.952 & 0.207 & 24.78 & 0.06 & 26.269 & -0.645 \\
\hline 56860.62 & WFC3-IR & F105W & 1159 & 2.899 & 0.167 & 25.11 & 0.06 & 26.269 & -0.645 \\
\hline 56886.63 & WFC3-IR & F105W & 1159 & 1.216 & 0.147 & 26.06 & 0.13 & 26.269 & -0.645 \\
\hline 56891.67 & WFC3-IR & F105W & 356 & 0.971 & 0.324 & 26.30 & 0.36 & 26.269 & -0.645 \\
\hline 56893.20 & WFC3-IR & F105W & 712 & 0.954 & 0.242 & 26.32 & 0.28 & 26.269 & -0.645 \\
\hline 56954.64 & WFC3-IR & F105W & 356 & 0.521 & 0.388 & 26.98 & 0.81 & 26.269 & -0.645 \\
\hline 56817.08 & WFC3-IR & F125W & 1206 & 8.459 & 0.191 & 23.91 & 0.02 & 26.230 & -0.901 \\
\hline 56833.15 & WFC3-IR & F125W & 756 & 7.753 & 0.255 & 24.01 & 0.04 & 26.230 & -0.901 \\
\hline 56841.83 & WFC3-IR & F125W & 806 & 6.015 & 0.227 & 24.28 & 0.04 & 26.230 & -0.901 \\
\hline 56850.07 & WFC3-IR & F125W & 806 & 4.343 & 0.224 & 24.64 & 0.06 & 26.230 & -0.901 \\
\hline 56891.86 & WFC3-IR & F140W & 712 & 2.578 & 0.344 & 25.42 & 0.14 & 26.452 & -1.076 \\
\hline 56893.06 & WFC3-IR & F140W & 712 & 3.026 & 0.363 & 25.25 & 0.13 & 26.452 & -1.076 \\
\hline 56955.58 & WFC3-IR & F140W & 1424 & 1.218 & 0.269 & 26.24 & 0.24 & 26.452 & -1.076 \\
\hline 56817.09 & WFC3-IR & F160W & 1206 & 4.831 & 0.263 & 24.24 & 0.06 & 25.946 & -1.251 \\
\hline 56833.21 & WFC3-IR & F160W & 756 & 3.965 & 0.241 & 24.45 & 0.07 & 25.946 & -1.251 \\
\hline 56841.84 & WFC3-IR & F160W & 756 & 3.011 & 0.234 & 24.75 & 0.08 & 25.946 & -1.251 \\
\hline 56850.08 & WFC3-IR & F160W & 756 & 2.744 & 0.223 & 24.85 & 0.09 & 25.946 & -1.251 \\
\hline 56860.67 & WFC3-IR & F160W & 1159 & 1.895 & 0.177 & 25.25 & 0.10 & 25.946 & -1.251 \\
\hline 56886.64 & WFC3-IR & F160W & 1159 & 1.677 & 0.191 & 25.38 & 0.12 & 25.946 & -1.251 \\
\hline 56812.0 & $\mathrm{ACS}$ & G800L & 3490 & $\cdots$ & $\cdots$ & $\cdots$ & $\cdots$ & $\ldots$ & $\ldots$ \\
\hline 56815.7 & ACS & G800L & 6086 & $\ldots$ & $\ldots$ & $\ldots$ & $\ldots$ & $\ldots$ & $\ldots$ \\
\hline
\end{tabular}

Notes.

${ }^{a}$ For non-positive flux values we report the magnitude as a $3 \sigma$ upper limit.

b Zero point difference: the magnitude shift for conversion from AB to Vega magnitude units. 
will see in Sections 4 and 5, both the spectroscopic and photometric data from the $\mathrm{SN}$ itself are consistent with the redshift of the fainter galaxy at $z=1.3457$, and incompatible with $z=1.742$ from this brighter galaxy. This means that the latter galaxy is a background object and therefore has no impact on the SN magnification.

The galaxy identified as the host is not close enough to the cluster core to necessarily be multiply imaged, but it is still possible that the host galaxy is one of the outer images of a multiple image system. In such a case, and given the position of the SN host, one would expect that another image of the galaxy would be present at a similar brightness, and would therefore be detectable in HST imaging. To date, no plausible candidate for a counter-image has been identified.

To measure the stellar mass of the host galaxy we use Equation (8) of Taylor et al. (2011), which relates the restframe $(g-i)$ color and $i$-band luminosity to the total stellar mass. To derive these values, we fixed the redshift at $z=1.3457$ and repeated the SED fitting using BPZ. From the best-fit SED we extracted rest-frame optical magnitudes, and corrected them for lensing using a magnification factor of $\mu=2.0-\mathrm{a}$ value that we will derive from the $\mathrm{SN}$ itself in Section 6. From this we determine the host galaxy mass to be $10^{9.8} M_{\odot}$.

\section{SPECTROSCOPY}

A spectrum of SN HFF14Tom was collected with the ACS G800L grism on 2014 June 4 and 7, when the SN was within 3 observer-frame days of the observed peak brightness in the F814W band. The observations - listed at the bottom of Table 2 - used 5 HST orbits from the FrontierSN program for a total spectroscopic exposure time of $\sim 10 \mathrm{ks}$. The grism data were processed and the target spectrum was extracted using a custom pipeline (Brammer et al. 2012), which was developed for the 3D-HST program (PI:Van Dokkum; PID:12177, 12328) and also used by the GLASS team.

Figure 2 shows the composite 1D ACS grism spectrum, combining all available G800L exposures, overlaid with SN model fits that will be described below. The spectrum is largely free of contamination, because the orientation was chosen to avoid nearby bright sources and the host galaxy is diffuse and optically faint. Thus, the SN spectral features can be unambiguously identified, most notably the red slope of the continuum and a prominent absorption feature at $\sim 8700 \AA$.

Spectra of Type I SNe (including all sub-classes Ia, Ib, and Ic) are dominated by broad absorption features, which cannot in general be used to directly extract a spectroscopic redshift (see, e.g., Filippenko 1997). As described below, we fit template spectra to the SN HFF14Tom data in two steps. First we determine a spectral classification - and get a preliminary estimate of the redshift and age-using the Supernova Identification (SNID) software (Blondin \& Tonry 2007). Second, we refine the redshift and age measurement using a custom Type Ia spectral template matching program.

\subsection{Classification with SNID}

The SNID program is designed to estimate the type, redshift, and age of a SN spectrum through cross-correlation matching with a library of template spectra, using the algorithm of Tonry \& Davis (1979). To account for possible distortions in the broad shape of the SN pseudo-continuum due to dust or instrumental calibration effects, SNID divides each SED by a
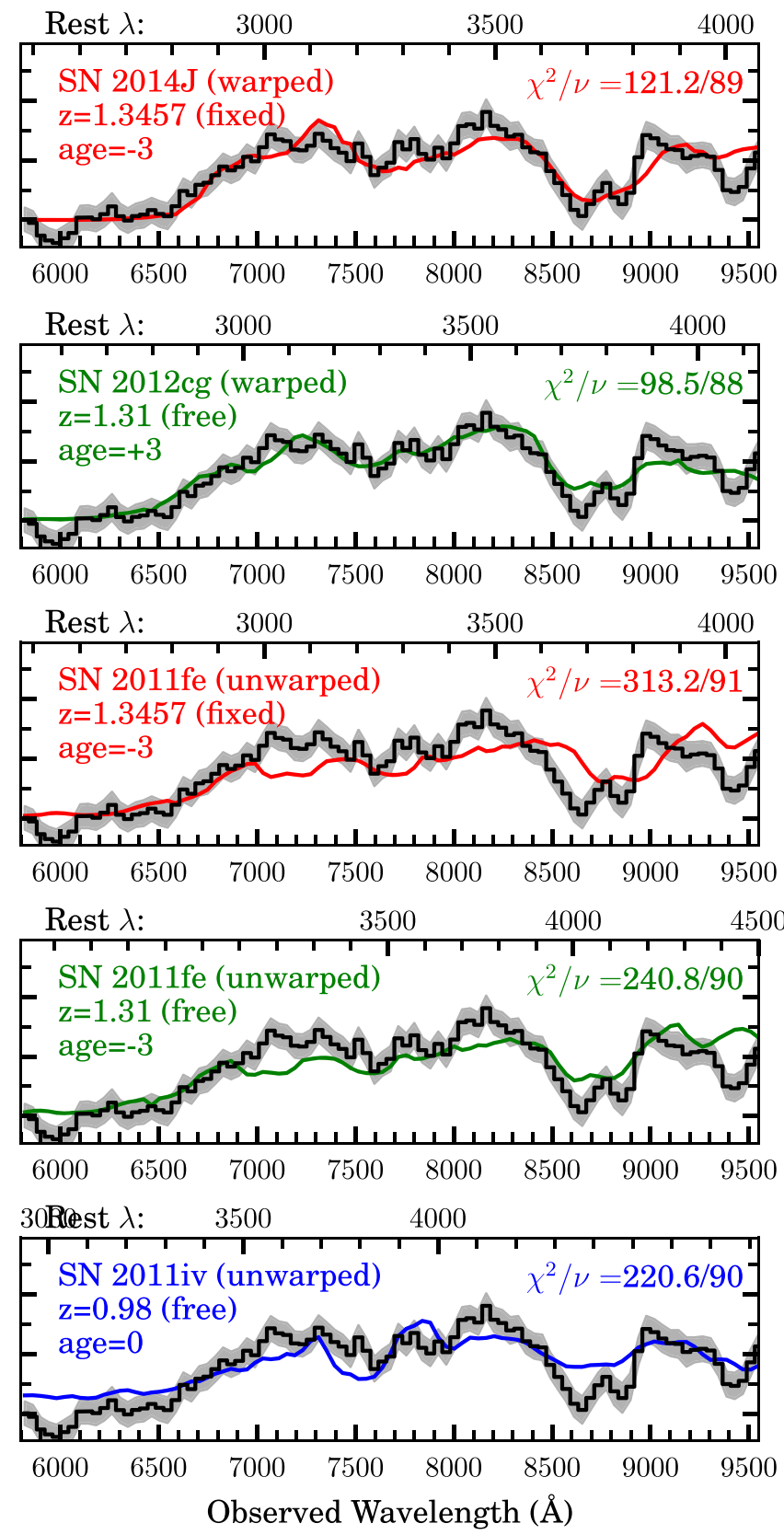

Figure 2. Redshift and age determination from spectral template matching to the the SN HFF14Tom maximum light spectrum. The $y$ axis plots flux in arbitrary units, and the $x$ axis marks wavelength in $\AA$ with the observer-frame on the bottom and rest-frame on the top. The HFF14Tom spectrum observed with the HST ACS G800L grism is shown in black, overlaid with model fits derived from a library of Type Ia templates that have extended rest-frame UV coverage. The top two panels show the best matching templates when using a smooth 3rd-order polynomial to warp the shape of the template pseudocontinuum, with the redshift fixed at $z=1.3457$ and then allowed to float as a free parameter. The lower three panels show matches found when the templates are not warped, both with and without fixing the redshift. The bottom panel shows the best match in this set, although at $z=0.98$ it is inconsistent with the host galaxy redshift prior and the light curve.

smooth cubic spline fit. This effectively removes the shape of the SN pseudo-continuum to leave behind a flat SED superimposed with spectral absorption and emission features. It is these features which drive the cross-correlation fit, so the SNID approach is insensitive to the overall color of the SED. We used v2.0 of the SNID template library, which includes 
template SEDs covering all Type Ia and core collapse subclasses, and has recently been updated with corrections and improvements to the Type $\mathrm{Ib} / \mathrm{c}$ templates (Liu \& Modjaz 2014).

In SNID the goodness of fit is evaluated primarily through the rlap parameter, which measures the degree of wavelength overlap and the strength of the cross-correlation peak. Typically, an rlap value $>5$ is required to be considered an acceptable match.

To match the SN HFF14Tom spectrum we use conservative constraints on age and redshift: limiting the age to \pm 5 restframe days from peak brightness and $0.8<z<1.8$, consistent with the SN light curve and the two plausible host galaxies. With these constraints we find that the only acceptable match is a normal Type Ia $\mathrm{SN}$ near $z=1.3$. The best match has rlap $=8.7$, using the normal Type Ia SN 2005cf at $z=1.35$ and age $=-2.2$ rest-frame days before peak. In contrast, the best non-Ia matches all have rlap $<2.5$.

Using SNID we can find an acceptable core collapse SN (CCSN) match only when we remove all age and redshift constraints. In this case the best non-Ia match is the Type Ic SN 1997ef, which delivers rlap $=6.8$ at $z=0.51$ and age $=47.3$ rest-frame days past peak (71 observer-frame days). This is not as good a fit as the best Type Ia models, is at odds with the host galaxy redshift prior, and is strongly disfavored by the shape and colors of the SN light curve (see Section 5).

From the preceding analysis, we conclude that HFF14Tom is a Type Ia $\mathrm{SN}$ at $z \approx 1.35$. At this redshift, the absorption at $\sim 8700 \AA$ corresponds to the blended Ca II H\&K and Si II $\lambda 3858$ features. Generally referred to as the $\mathrm{Ca} H \& \mathrm{~K}$ feature, this absorption is commonly seen in Type Ia SN spectra near maximum light, although it is also prominent in the spectra of Type Ib and Ic CC SNe. The red color of the HFF14Tom SED is qualitatively consistent with a redshift of $z>1$-although this information was not used by SNID for the template matching. As we will see in Section 5, this spectral classification of SN HFF14Tom is reinforced by the photometric information, which also supports classification as a Type Ia $\mathrm{SN}$ at $z \approx 1.35$.

\subsection{Spectral Fitting with UV Type Ia Templates}

To refine the redshift and phase constraints on HFF14Tom, we next fit the spectrum with a custom spectral matching program that employs a library of Type Ia SN SEDs. This library is similar to the Type Ia spectral set used by SNID, but also includes more recent $\mathrm{SNe}$ with well-observed spectral time series that extend to rest-frame UV wavelengths (e.g., SN 2011fe and 2014J). We first use an approach similar to the SNID algorithm: warping the pseudo-continuum of each template spectrum by dividing out a third-order polynomial to match the observed SED of SN HFF14Tom. This approach will find templates that have similar abundances and photospheric velocities. With the redshift fixed at $z=1.3457$ we find the best fit is a spectrum from the normal Type Ia SN 2014J (Foley et al. 2014), with a $\chi^{2}$ per degree of freedom $\nu$ of $\chi^{2} / \nu=121.8 / 89$ shown in the top panel of Figure 2. The excess variance in this fit may be attributed to the intrinsic variation of Type Ia SN spectra, which is more prominent at UV wavelengths (e.g., Foley et al. 2008; Wang et al. 2012) and is not fully represented in the available template library. Allowing the redshift as an additional free parameter, we still find results that are consistent with the SNID fits and $<2 \sigma$ from the host galaxy redshift: $z=1.31 \pm 0.02$ and a phase of $0 \pm 3$ rest-frame days. The best-fitting spectral template in this case is the normal Type Ia SN 2012cg (Amanullah et al. 2015) at $z=1.31$ with $\chi^{2} / \nu=98.5 / 88$ (second panel of Figure 2).

Next, we repeat the fitting, but without any warping of the templates to account for differences in the continuum shape. In this iteration we only allow each template SED to be scaled in flux coherently at all wavelengths, so the fits are more sensitive to the overall color of the SED. ${ }^{39}$ Fixing the redshift to $z=$ 1.3457 , we find the best match is from the normal Type Ia SN $2011 \mathrm{fe}$ (Mazzali et al. 2014), though the fit is quite poor, with $\chi^{2} / \nu=313.2 / 91$ (third panel of Figure 2). When the redshift is allowed as a free parameter the HFF14Tom SED is still matched best by a SN 2011fe template, now at redshift $z=1.31$ with $\chi^{2} / \nu=240.8 / 90$ (fourth panel of Figure 2). SN 2011fe had effectively no dust reddening (e.g., Li et al. 2011a; Nugent et al. 2011), so the fact that SN $2011 \mathrm{fe}$ provides the best un-warped template match is further evidence that $\mathrm{SN}$ HFF14Tom suffers from very little dust extinction.

Without the continuum warping, an alternative fit also arises: the fast-declining (91bg-like) Type Ia SN 2011iv (Foley et al. 2012) at $z=0.98 \pm 0.01$. Formally, this match provides a slightly better fit to the unwarped HFF14Tom spectrum $\left(\chi^{2} / \nu=220.6 / 90\right.$, bottom panel of Figure 2$)$, although the fit is notably poorer at $\sim 8700 \AA$ where the most significant absorption feature is found. Furthermore, a redshift $z \sim 1$ is at odds with the spectroscopic redshift of the nearest galaxy $(z=1.3457)$, and we will see in the following section that the photometric data is also incompatible with a Type Ia $\mathrm{SN}$ at $z \sim 1.0$.

Setting aside the $z \sim 1$ solution, all other template matches provide a consistent redshift constraint of $z=1.31 \pm 0.02$, regardless of whether the templates are warped to match the SN HFF14Tom continuum shape. The inferred age from these fits is $0 \pm 3$ rest-frame days from peak brightness, which is also consistent with the observed light curve. Taken together with the host galaxy spectroscopic redshift, these fits suggest that $\mathrm{SN}$ HFF14Tom is a normal Type Ia SN at $z=1.3457$ with an SED color close to SN 2011fe, but spectral absorption features similar to SN 2014J.

\section{PHOTOMETRIC CLASSIFICATION}

Relative to other $\mathrm{SNe}$ at $z>1$, the SN HFF14Tom light curve was unusually well sampled at rest-frame ultraviolet wavelengths, due to the rapid cadence of the HFF imaging campaign. These ACS observations therefore provide a tight constraint on the time of peak brightness and the evolution of the SN color. Supplemental observations with the WFC3-IR camera provided critical rest-frame optical photometry, enabling a measurement of the apparent luminosity distance through light curve fitting.

As a check on the spectral classification of SN HFF14Tom (Section 4.1), we independently classified the SN using a Bayesian photometric classifier. We use the sncosmo software package ${ }^{40}$ to simulate $\mathrm{SN}$ light curves from $z=0.3$ to 2.3 and evaluate the classification probability using traditional Bayesian model selection (as in Jones et al. 2013;

\footnotetext{
39 Note that gravitational lensing does not affect the color of background sources at all, so the expected lensing magnification of HFF14Tom does not affect this analysis.

${ }^{40}$ http://sncosmo.github.io/
} 
Graur et al. 2014; Rodney et al. 2014, 2015). In this analysis we represent normal Type Ia SNe with the SALT2 model (Guy et al. 2010), and CC SNe with 42 discrete templates (26 Type II and 16 Type $\mathrm{Ib} / \mathrm{c}$ ) drawn from the template library of the Supernova Analysis software package (SNANA, Kessler et al. 2009b). ${ }^{41}$ Likelihoods are defined by comparing the observed fluxes to model predictions in all passbands where the model is defined. In practice, this means we exclude the SN detections in the F435W and F606W bands, which are too blue for our models at $z>0.85$.

The CC SN models have free parameters for date of peak brightness $\left(t_{\mathrm{pk}}\right)$, amplitude, and redshift (z). Due to the expected impact of gravitational lensing magnification, we use a flat prior for the intrinsic luminosity of all SN sub-classes. We also apply a flat prior for the SN redshift, which allows our photometric analysis to provide an independent check on the host galaxy photo- $z$ and spectroscopic redshift (Sections 3 and 4.2).

For Type Ia SNe the SALT2 model has two additional parameters that control the shape $\left(x_{1}\right)$ and color $(c)$ of the light curve. We use conservative priors here, defined to encompass a range of Type Ia SN shapes and colors that is broader than typically allowed in cosmological analyses (see e.g., Kessler et al. 2009a; Sullivan et al. 2011; Rest et al. 2014). For $x_{1}$ the prior is a bifurcated Gaussian distribution with mean $\bar{x}_{1}=0$, dispersion $\sigma_{x_{1}}^{+}=0.9$ and $\sigma_{x_{1}}^{-}=-1.5$. The bifurcated Gaussian prior for the color parameter $c$ has $\bar{c}=0.0, \sigma_{c}^{-}=0.08$, and $\sigma_{c}^{+}=0.54$. The $c$ parameter in SALT2 combines intrinsic SN color and extinction due to dust, so the large red tail of this distribution allows for the possibility of several magnitudes of dust extinction along the HFF14Tom line of sight.

We also assign a class prior for each of the three primary SN sub-classes (Type Ia, Ib/c, and II), using a fixed relative fraction for each sub-class as determined at $z=0$ by Smartt et al. (2009) and $\mathrm{Li}$ et al. (2011b). A more rigorous classification would extrapolate these local SN class fractions to higher redshift using models or measurements of the volumetric SN rate. For simplicity, we do not vary the class priors with redshift, and in practice these priors do not have any significant impact on the resulting classification.

The final photometric classification probability for SN HFF14Tom is $p(\mathrm{Ia} \mid \boldsymbol{D})=1.0$, with the classification probability from all CCSN sub-classes totaling less than $10^{-32}$. Although this Bayesian classification utilizes the full posterior probability distribution, for illustration we highlight in Figure 3 a single best-fit model for each sub-class. This demonstrates how the CC SN models fail to adequately match the observed photometry. In particular, only the Type Ia model can simultaneously provide an acceptable fit to the wellsampled rising light curve in F814W and the F814W-F160W color near peak.

The marginal posterior distribution in redshift for the Type Ia model is sharply peaked at $z=1.35 \pm 0.02$, which is fully consistent with the redshift of the presumed host galaxy $(z=1.3457)$ as well as the redshift of $z=1.31 \pm 0.02$ derived from the SN spectrum in Section 4.2. The time of peak brightness is also tightly constrained at $t_{\mathrm{pk}}=56816.3 \pm 0.3$, which means the $H S T$ grism observations were collected within 2 rest-frame days of the epoch of peak brightness-also consistent with our spectroscopic analysis.

\footnotetext{
${ }^{41}$ Throughout this work we use SNANA v10_35g.
}
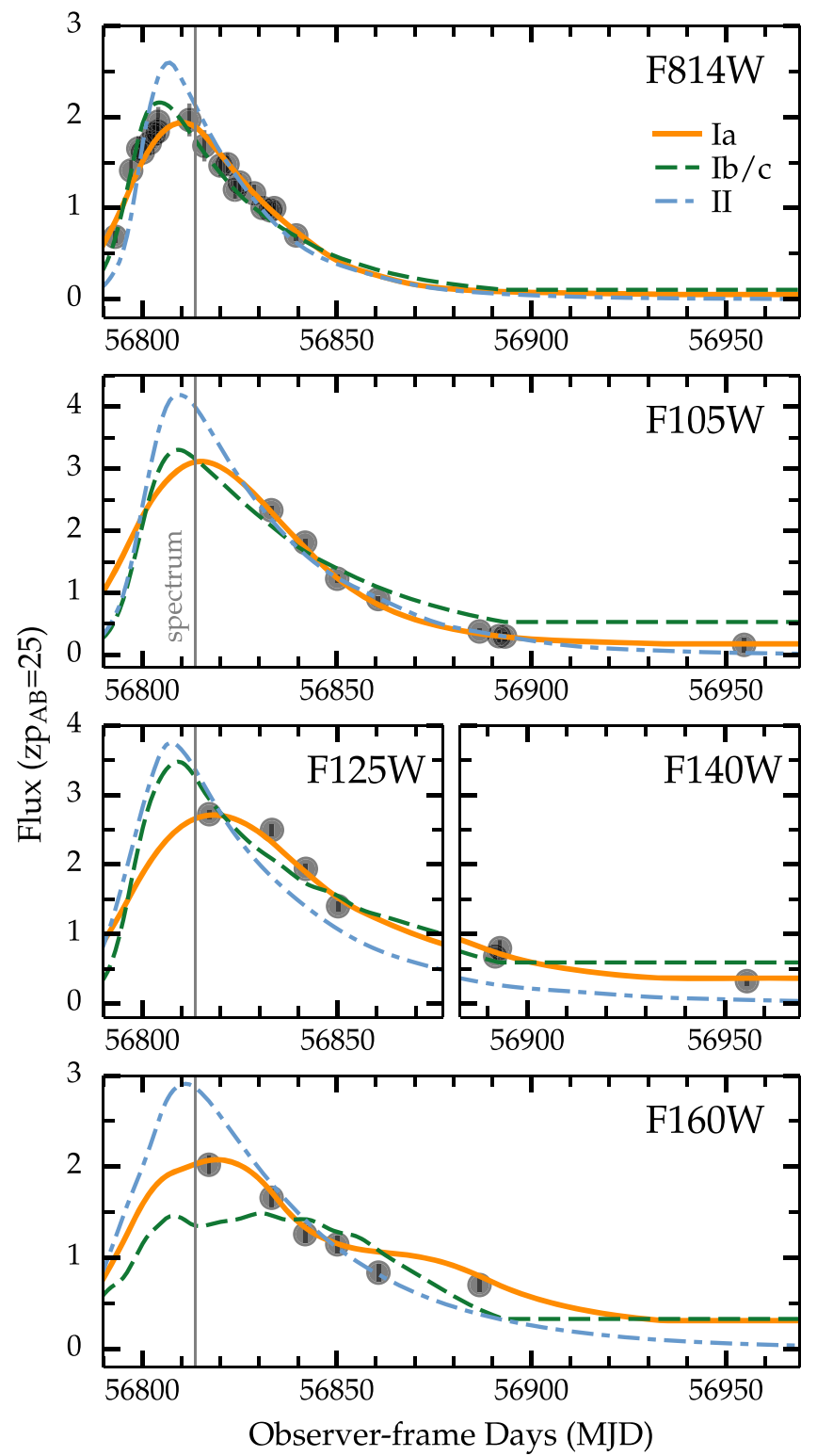

Figure 3. Maximum likelihood model for each SN sub-class, derived from Bayesian model selection using the photometric data alone. Gray points show the observed SN HFF14Tom photometry with error bars, though these are typically smaller than the size of the marker. The Type Ia model (orange solid line) is drawn from the SALT2 template at $z=1.35$. The best match from all Type Ib/Ic models is based on the SN Ic SDSS-14475 at $z=0.695$ (green dashed line). For the Type II class, the best match is from the Type II-L SN 2007pg at $z=1.8$ (blue dash-dot line). The Type Ia model is by far the best match, and the only one that is consistent with both the spectroscopic redshift of the probable host galaxy and the spectroscopic redshift from the SN spectrum. The date of the $H S T$ spectral observations is marked with a thin gray vertical line.

\section{DISTANCE MODULUS AND MAGNIFICATION}

With the type and redshift securely defined as a normal Type Ia SN at $z=1.3457$, we now turn to fitting the light curve with Type Ia templates to measure the distance modulus (Section 6.1) and then derive the gravitational lensing magnification (Section 6.2). In this process, we would like to avoid introducing systematic uncertainties inherent to any assumed cosmological model. To that end, in Section 6.2 we follow P14 and define the magnification by comparing the measured distance modulus of SN HFF14Tom against an 


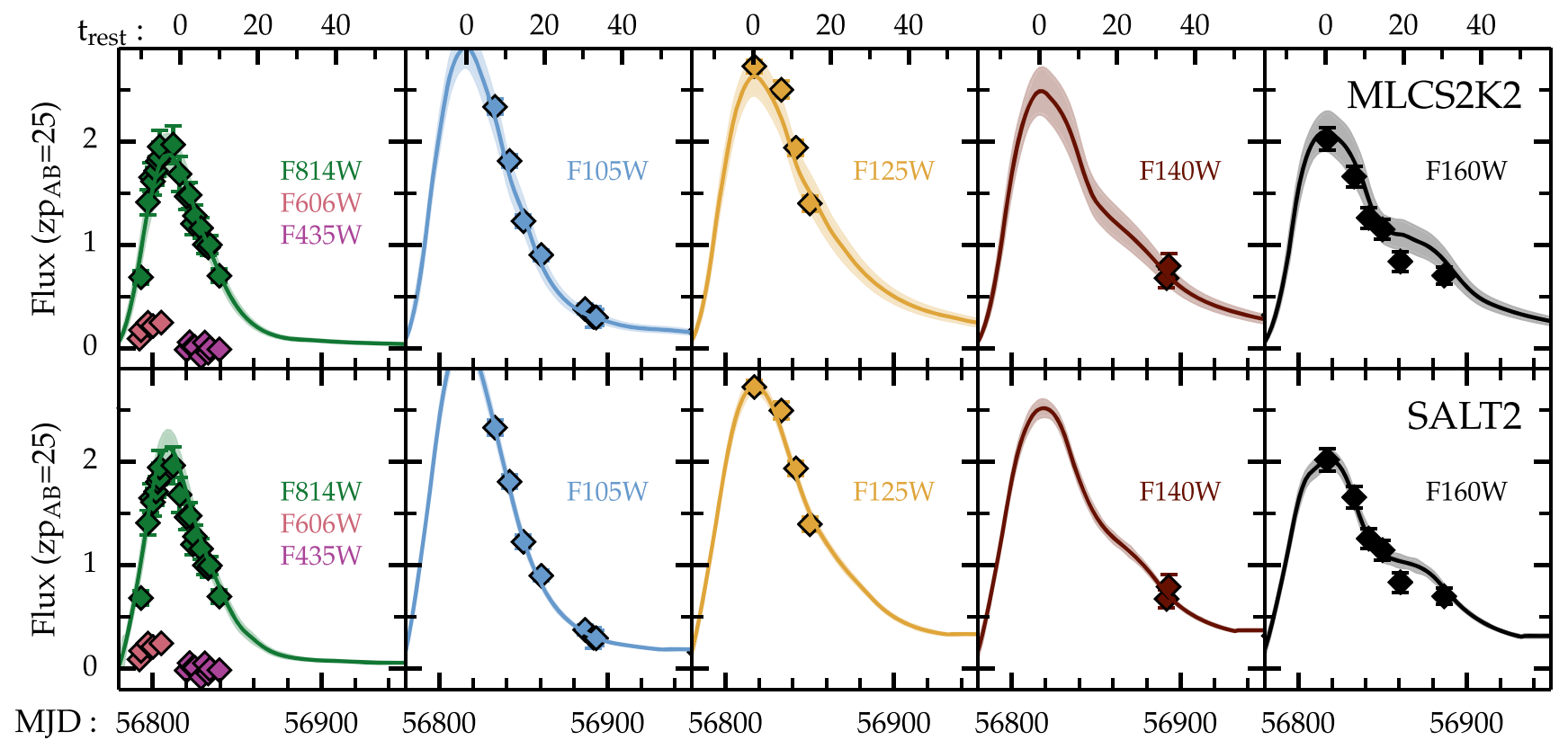

Figure 4. Type Ia light curve fits to SN HFF14Tom using the MLCS2k2 (top row) and SALT2 (bottom row) fitters. The model redshifts are set to $z=1.3457$ as determined from combined spectroscopic and photometric constraints. Solid lines denote the best-fit model and shaded lines show the range allowed by $1 \sigma$ uncertainties on the model parameters. Observed fluxes are shown as diamonds, scaled to an $\mathrm{AB}$ magnitude zero point of 25. Error bars are plotted, but most are commensurate with the size of the points. The left-most panel includes observations in the F435W and F606W filters, although these were not used for the fit, as they are bluer than the minimum wavelength for the model. The lower axis marks time in observer frame days, while the top axis shows the time in the rest frame relative to the epoch of peak brightness.

average distance modulus derived from a "control sample" of unlensed Type Ia $\mathrm{SNe}$ at similar redshift. This allows us to make only the minimal assumption that the redshift-distance relationship for Type Ia $\mathrm{SN}$ is smooth and approximately linear over a small redshift span, which should be true for any plausible cosmological model.

\subsection{Light Curve Fitting}

As in P14, we derive a distance modulus for SN HFF14Tom and all $\mathrm{SNe}$ in our control sample using two independent light curve fitters: the SALT2 model described above and the MLCS2k2 model (Jha et al. 2007). The best-fit light curves from both models are shown in Figure 4 . With both fitters we find light curve shape and color parameters for HFF14Tom that are fully consistent with a normal Type Ia SN. For SALT2, with the redshift fixed at $z=1.3457$, we find a light curve shape parameter of $x_{1}=0.135 \pm 0.199$ and a color parameter of $c=-0.127 \pm 0.025$, yielding a $\chi^{2}$ value of 45.0 for 36 degrees of freedom, $\nu$. With the MLCS2k2 fitter the best-fit shape parameter is $\Delta=-0.082 \pm 0.070$ and the color term is $A_{V}=0.011 \pm 0.025$, giving $\chi^{2} / \nu=22.8 / 36$.

The MLCS2k2 fitter returns a distance modulus ${ }^{42} \mathrm{dm}_{\text {MLCS2k } 2}$ directly, as it is defined to be one of the free parameters in the model. To derive dm from the SALT2 fit, we use

$$
\mathrm{dm}_{\mathrm{SALT} 2}=m_{B}^{*}-M+\alpha(s-1)-\beta C .
$$

Here the parameters for light curve shape $s$ and color $C$ correspond to the SiFTO light curve fitter (Conley et al. 2008), so we first use the formulae from Guy et al. (2010) to convert

\footnotetext{
42 We use "dm" to indicate the distance modulus to avoid confusion, reserving the symbol $\mu$ to refer to the lensing magnification. This " $\mathrm{dm}$ " is a standard distance modulus, defined as $\mathrm{dm}=5 \log _{10} d_{\mathrm{L}}+25$, where $d_{\mathrm{L}}$ is the luminosity distance in Mpc.
}

from SALT2 $\left(x_{1}\right.$ and $\left.c\right)$ into the equivalent SiFTO parameters. We also add an offset of $0.27 \mathrm{mag}$ to the value of $m_{B}^{*}$ returned by SNANA, in order to match the arbitrary normalization of the SALT2 fitter used by Guy et al. (2010) and Sullivan et al. (2011). This conversion from SALT2 to SiFTO is necessary, as it allows us to adopt values for the constants $M, \alpha$, and $\beta$ from Sullivan et al. (2011), which have been calibrated using $472 \mathrm{SNe}$ from the SNLS3 sample (Conley et al. 2011): $M=-19.12 \pm 0.03, \alpha=1.367 \pm 0.086$, and $\beta=$ $3.179 \pm 0.101$.

The SNANA version of the MLCS2k2 fitter returns a value for the distance modulus $\left(\mathrm{dm}_{\mathrm{MLCS} 2 \mathrm{k} 2}\right)$ that has an arbitrary zero point offset relative to the SALT2 distances $\left(\mathrm{dm}_{\mathrm{SALT} 2}\right)$. To put the two distances onto the same reference frame we add a zeropoint correction of 0.20 mag to the MLCS2k2 distances as in P14. This correction was derived by applying both fitters to a sample of Type Ia SNe from the SDSS survey (Holtzman et al. 2008; Kessler et al. 2009a), with the extinction law $R_{V}$ fixed at 1.9 .

The total uncertainty in the distance modulus is

$$
\sigma_{\mathrm{tot}}=\sqrt{\sigma_{\text {stat }}^{2}+\sigma_{\mathrm{int}}^{2}} .
$$

The $\sigma_{\text {stat }}$ term is the statistical uncertainty, which encapsulates uncertainties from the data and the model, and $\sigma_{\text {int }}$ accounts for the remaining unmodeled scatter. This latter term is derived by finding the amount of additional distance modulus scatter that needs to be added to a Type Ia SN population to get $\chi^{2}$ per degree of freedom equal to 1 for a fiducial cosmological model fit to the Type Ia SN Hubble diagram (distance modulus versus redshift). Thus, $\sigma_{\text {int }}$ is designed to account for any unknown sources of scatter in the Type Ia SN population, including unidentified errors in the data analysis as well as the natural scatter in intrinsic Type Ia SN luminosities. The value 
Table 3

HFF14Tom Measured Distance Modulus and Magnification at $z=1.3457$

\begin{tabular}{lccc}
\hline \hline & \multicolumn{2}{c}{ Distance Modulus } & Measured \\
Fitter & HFF14Tom & Control & Magnification \\
\hline MLCS2k2 & $44.205 \pm 0.12$ & $44.97 \pm 0.06$ & $2.03 \pm 0.29$ \\
SALT2 & $44.177 \pm 0.18$ & $44.92 \pm 0.06$ & $1.99 \pm 0.38$ \\
\hline
\end{tabular}

of $\sigma_{\text {int }}$ may be expected to vary as a function of redshift and also from survey to survey.

Recent work has highlighted the inadequacy of this simplistic approach for handling intrinsic scatter in the Type Ia SN population (Marriner et al. 2011; Kessler et al. 2013; Betoule et al. 2014; Mosher et al. 2014; Scolnic et al. 2014b), but a full consideration of those alternative approaches is beyond the scope of this work. We adopt the simple approach of using a single empirically defined value for $\sigma_{\text {int }}$, reflecting principally an intrinsic scatter in Type Ia $\mathrm{SN}$ intrinsic luminosity that is fixed across time and phase. Measurements of $\sigma_{\text {int }}$ range from 0.08 mag (Jha et al. 2007; Conley et al. 2011) to $0.15 \mathrm{mag}$ (Kessler et al. 2009a; Suzuki et al. 2012). We adopt a value of $\sigma_{\text {int }}=0.08 \mathrm{mag}$, as derived by Jha et al. (2007) and Conley et al. (2011). Although this is on the low end of the range reported in the literature, this value is the most appropriate to apply to our analysis for two reasons. First and foremost, for the SALT2 fitter we are using light curve fit parameters $(\alpha, \beta)$ with associated uncertainties that have been derived from the joint analysis of Conley et al. (2011) and Sullivan et al. (2011). Similarly, the implementation of MLCS2k2 that we have used is based on the uncertainty model derived from model training in Jha et al. (2007). Inflating $\sigma_{\text {int }}$ beyond $0.08 \mathrm{mag}$ would therefore be equivalent to driving the reduced $\chi^{2}$ of the Type Ia SN Hubble diagram to $<1$. Second, the value of $0.08 \mathrm{mag}$ determined in Conley et al. (2011) is specific to the HST SN sample, from Riess et al. (2007) and Suzuki et al. (2012), which is the SN subset that is most similar to HFF14Tom in terms of redshift and data analysis. Larger values for $\sigma_{\text {int }}$ are typically associated with SN samples at significantly lower redshifts that have less homogeneous data collection and analysis.

Final values for the $\mathrm{SN}$ distance modulus are shown in Table 3, adopting the spectroscopic redshift $(z=1.3457)$. In addition to modifying the distance modulus uncertainty for SN HFF14Tom, we also add $\sigma_{\text {int }}=0.08 \mathrm{mag}$ in quadrature to the uncertainty for every $\mathrm{SN}$ in the control sample. Note however that the uncertainty on the control sample value at $z=1.3457$ is only $0.06 \mathrm{mag}$, smaller than the intrinsic dispersion of any single SN because it reflects our measurement error on the mean distance modulus of the population. The distance moduli derived from the SALT2 and MLCS2k2 light curve fitters are fully consistent within the uncertainties.

\subsubsection{Host Galaxy Mass Correction}

It is now an accepted practice in cosmological analyses using Type Ia SN to apply a correction to the luminosity of each SN based on the stellar mass of its host galaxy. Typically this is described as a simple bifurcation of the SN population: $\mathrm{SNe}$ that appear in more massive hosts are observed to be $\sim 0.08$ mag brighter (after corrections for light curve shape and color) than SNe in low-mass hosts (Kelly et al. 2010; Sullivan et al. 2010). The dividing line for this purely empirical "mass step" correction is generally set around $10^{10} M_{\odot}$. Although this threshold value is somewhat arbitrary (see, e.g., Betoule et al. 2014), it happens to be very close to the SN HFF14Tom host galaxy mass of $10^{9.8} M_{\odot}$ (Section 3 ).

The physical mechanism that drives the mass step is not yet understood, but may be related to the metallicity or age of the SN progenitor systems. In either case, the significance of this effect should decrease with redshift, as metal-rich passive galaxies become much less common at $z>1$ (see e.g., Rigault et al. 2013; Childress et al. 2014). Indeed, when the size of the mass step correction is allowed to vary with redshift, there is no significant evidence that a non-zero mass step is required at $z>1$ (Suzuki et al. 2012; Betoule et al. 2014; Shafer \& Huterer 2014). Given the absence of a clear physical model and the lack of empirical support for a high-z mass step, we do not apply any correction to SN HFF14Tom or the control sample.

If we were to apply the correction to this sample using the standard approach, then the SN HFF14Tom distance modulus would not be adjusted, because its host galaxy mass (Section 3) is just below $10^{10} M_{\odot}$. Note that there is some circularity here, as we used the SN magnification in deriving the host galaxy mass, and now use the host mass to inform the magnification. However, regardless of whether the HFF14Tom host mass falls above the mass threshold or below, this correction is not substantial enough to account for the observed magnification tension. The appropriate correction to be applied to a $\mathrm{SN}$ above the mass threshold in this redshift range would be $<0.04$ mag (Rigault et al. 2013; Childress et al. 2014), which results in a change to the inferred magnification of HFF14Tom that is much less than the observed discrepancy of $\sim 0.24$ mag.

\subsection{Control Sample Comparison}

The unlensed sample comprises 22 spectroscopically confirmed Type Ia SNe in the range $1.1<z<1.6$ from three $H S T$ surveys: 11 from the GOODS Higher- $z$ SN search $^{43}$ (Strolger et al. 2004; Riess et al. 2007), 7 from $\mathrm{SCP}^{44}$ (Suzuki et al. 2012), and 4 from CANDELS ${ }^{45}$ (Rodney et al. 2012, 2014). Using the SALT2 and MLCS2k2 fitters as described above, we get distance modulus measures for every object in this control sample. We then fit a linear relationship for distance modulus versus redshift, and derive a prediction for the distance modulus of a normal Type Ia SN at the redshift of SN HFF14Tom (Figure 5). This predicted value is given in Table 3 under the "Control" column. The difference between the observed distance of SN HFF14Tom and this control sample value is attributed to the magnification from gravitational lensing:

$$
\mathrm{dm}_{\text {control }}-\mathrm{dm}_{\text {HFF14Tom }}=2.5 \log _{10} \mu .
$$

The inferred magnifications for the two different fitters are reported in the final column of Table 3. Although we have chosen to use a cosmology-independent approach to determine the magnifications, we note that these results are fully consistent with the values that would be determined by comparing the observed SN HFF14Tom distance modulus against the predicted value from a flat $\Lambda$ CDM cosmology. Adopting the cosmological parameters used in P14 and derived

\footnotetext{
43 GOODS: the Great Observatories Origins Deep Survey, PI:Giavalisco, HST-PID:9425, 9583.

44 SCP: the Supernova Cosmology Project, PI:Perlmutter.

45 CANDELS: the Cosmic Assembly Near-infrared Deep Extragalactic Legacy Survey, PI:Faber \& Ferguson.
} 


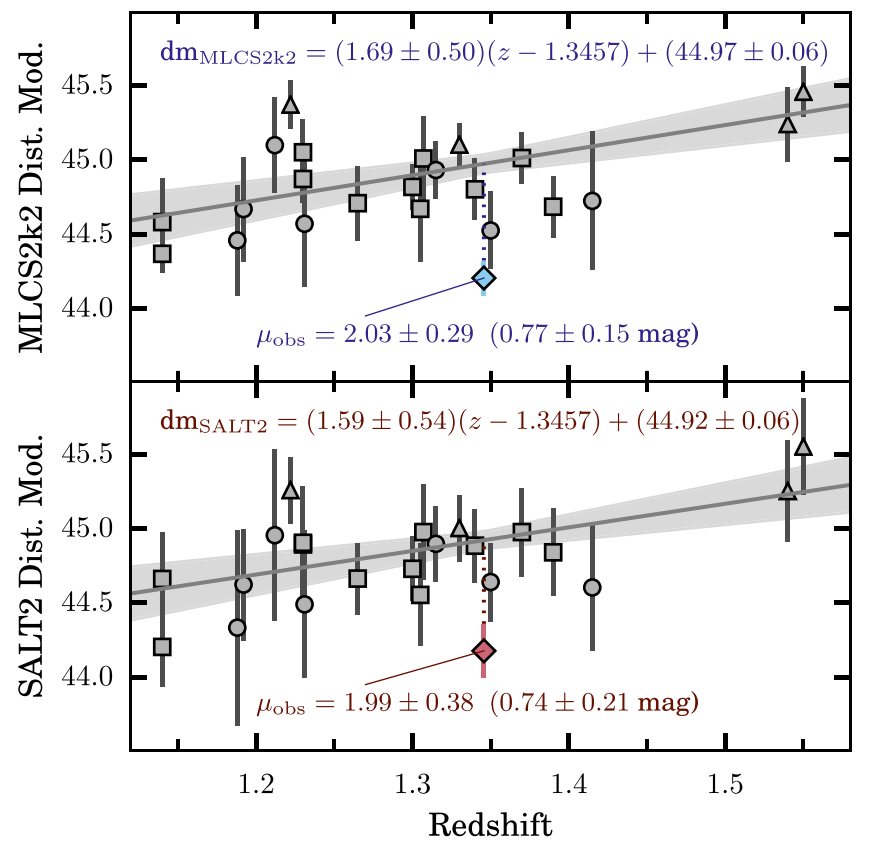

Figure 5. Measurement of the lensing magnification from comparison of the HFF14Tom distance modulus to a sample of unlensed field SN. SNe from the GOODS program are plotted as squares, those from the SCP survey are shown as circles, and the CANDELS objects are triangles. The distance modulus for each $\mathrm{SN}$ is derived from light curve fits using the MLCS2k2 fitter (top panel) and the SALT2 fitter (bottom panel). Gray lines with shading show a linear fit to the unlensed control sample is shown, anchored at the redshift of SN HFF14Tom, with fit parameters given at the top. The derived magnification is reported at the bottom of each panel.

in Sullivan et al. (2011) $\left(H_{0}=71.6, \Omega_{\mathrm{m}}=0.27, \Omega_{\Lambda}=0.73\right)$ would give a predicted distance modulus $\mathrm{dm}_{\Lambda \mathrm{CDM}}=44.90$ and a measured magnification $\mu=1.9$. Substituting alternative cosmological parameters (e.g., Betoule et al. 2014; Planck Collaboration et al. 2015) would not significantly change the inferred magnification or affect our conclusions.

\section{COMPARISON TO MODEL PREDICTIONS}

Before the Frontier Fields observations began, STScI issued a call for lens modeling teams to generate mass models of all six Frontier Field clusters, using a shared collection of all imaging and spectroscopic data available at the time. In response to this opportunity, five teams generated seven models for Abell 2744. These models necessarily relied on pre-HFF data, and were required to be complete before the HFF program began, in order to enable the estimation of magnifications for any new lensed background sources revealed by the HFF imaging. An interactive web tool was created by D. Coe and hosted at STScI, to extract magnification estimates and uncertainties from each model for any given redshift and position. In this work we also consider eight additional models that were created later. Some of these are updates of the original models produced in response to the lens modeling call, and some of them include new multiply imaged galaxies discovered in the HFF imaging as well as new redshifts for lensed background galaxies. Magnifications derived from several of these later models are also available on the interactive web tool hosted by STScI. The full list of models and details on their construction are given in Table 4.

Table 5 gives each model's predicted magnification and uncertainty for a source at $z=1.3457$ and at the position of
HFF14Tom. For thirteen of these models the SN magnification qualifies as a true "blind test," as they were completed before the SN HFF14Tom magnification measurement was known. The final four models (Bradac-v2, Lam-v2, CATSv2.1 and 2.2) are technically not blind, although none of these modelers used the SN magnification as a constraint, and the modelers did not have access to the final $\mathrm{SN}$ magnification value when constructing their model.

These models represent a broad sampling of the techniques and assumptions that can be applied to the modeling of mass distributions in galaxy clusters. We discuss two primary modeling choices here, but a more rigorous comparison of these diverse lens modeling techniques is beyond the scope of this work. For a complete discussion of each model's methodology the reader is directed to the listed references.

(1) Parametric versus Free-form: Ten of these models are so-called parametric ${ }^{46}$ models, and the remaining seven are free-form models. Broadly speaking, the parametric models use parameterized density distributions to describe the arrangement of mass within the cluster. Therefore, parametric models rely (to varying degrees) on the assumption that the cluster's dark matter can be described by analytic forms such as NFW halos (Navarro et al. 1997), or pseudo isothermal elliptical mass distributions (PIEMD; Kassiola \& Kovner 1993).

The seven free-form models divide the cluster field into a grid, generally using a multi-scale grid to get better sampling in regions with a higher density of information (e.g., density of multiple images). Each grid cell is assigned a mass or a potential, and then the mass values are iteratively refined to match the observed lensing constraints. In some cases an adaptive grid is used so that the grid spacing itself can also be modified as the model is iterated (e.g., Liesenborgs et al. 2006; Bradač et al. 2009; Merten et al. 2009).

As usual, there is a tradeoff between a model's flexibility, the strength of the model assumptions, and the resulting uncertainties. In a probabilistic framework, the posterior distribution function of the desired quantities depends on all the priors, including model assumptions like parametrization. In general, free-form methods tend to be more flexibile than simply parameterized models, and thus tend to result in larger error bars. In brief, if the free form methods are too flexible, then they will result in overestimated error bars. Conversely, if the simply parameterized models are too inflexible, they will result in underestimated error bars.

(2) Strong versus Strong + Weak: Thirteen of the mass models evaluated here are constrained only using stronglensing features such as multiply imaged background galaxies and highly magnified arcs. There are now $\sim 150$ known lensed images behind Abell 2744 (Jauzac et al. 2015), but SN HFF14Tom is located several arcseconds outside the core region of the cluster where these multiply imaged galaxies are found. Therefore these "strong-lensing only" models must necessarily rely on extrapolations to provide a prediction for the SN HFF14Tom magnification.

The other four models in our comparison set also use the strong lensing constraints from the core region, but additionaly use weak lensing measurements to provide additional constraints on the mass distribution farther from the cluster core.

\footnotetext{
46 Although this nomenclature is becoming standard in the literature, it is somewhat misleading, as the pixels or grid cells in free-form models are effectively parameters as well. Perhaps "simply parameterized" would be more accurate, though we adopt the more common usage here.
} 
Table 4

Tested Lens Models for Abell 2744

\begin{tabular}{|c|c|c|c|c|c|c|}
\hline Model & $N_{\text {sys }}{ }^{\mathrm{a}}$ & $N_{\text {im }}{ }^{\mathrm{b}}$ & $N_{\text {spec }}{ }^{\mathrm{c}}$ & $N_{\text {phot }}{ }^{\mathrm{d}}$ & References & Description \\
\hline$\overline{\operatorname{Bradac}(\mathrm{v} 1)^{\mathrm{e}}}$ & 16 & 56 & 2 & 14 & HFF $^{\text {f }}$ Bradač et al. (2009) & SWUnited ${ }^{\mathrm{g}}$ : Free-form, strong+weak-lensing based model.Errors from bootstrap resampling only weak-lensing constraints. \\
\hline CATS(v1) & 17 & 60 & 2 & 14 & HFF; Richard et al. (2014) & CATS $^{\text {h }}$ team implementation of LENSTOOL $\mathrm{L}^{\mathrm{i}}$ parametric strong-lensing based model. \\
\hline CATS(v1.1) & 17 & 60 & 2 & 14 & Richard et al. (2014) & CATS team LENSTOOL parametric model with both strong and weak lensing constraints. \\
\hline Merten $^{\mathrm{e}}$ & 16 & 56 & 2 & 14 & HFF; Merten et al. (2011) & SaWLENS, ${ }^{\mathrm{k}}$ Grid-based free-form strong+weak lensing based model using adaptive mesh refinement. \\
\hline Sharon(v1) & 17 & 60 & 2 & 14 & HFF; & LENSTOOL parametric strong-lensing based model \\
\hline Sharon(v2) & 15 & 47 & 3 & 11 & Johnson et al. (2014) & LENSTOOL parametric strong-lensing based model. Includes cosmological parameter variations in uncertainty estimates. \\
\hline Zitrin-LTM & 10 & 44 & 2 & 0 & HFF; Zitrin et al. (2009) & Parametric strong-lensing model, adopts the Light-traces-mass assumption for both the luminous and dark matter. \\
\hline Zitrin-NFW & 10 & 44 & 2 & 0 & HFF; Zitrin et al. (2013) & Parametric strong-lensing model using PIEMD profiles for galaxies and NFW profiles for dark matter halos. \\
\hline Williams & 10 & 40 & 2 & 8 & HFF & GRALE $^{1}$ : Free-form strong-lensing model using a genetic algorithm. \\
\hline \multicolumn{7}{|r|}{ Post-HFF Models: Include Data from the HFF Program } \\
\hline CATS(v2) & 50 & 151 & 4 & 1 & Jauzac et al. (2015) & Updated version of the CATSv1 model, adds 33 new multiply imaged galaxies, for a total of 159 individual lensed images. \\
\hline Diego $^{\mathrm{m}}$ & 15 & 48 & 4 & 11 & Diego (2014) & WSLAP ${ }^{\mathrm{n}}$ : Free-form strong-lensing model using a grid-based method, supplemented by deflections fixed to cluster member galaxies. \\
\hline GLAFIC & 24 & 67 & 3 & 12 & Ishigaki et al. (2015) & Parametric strong-lensing model using v1.0 of the GLAFIC code. ${ }^{\circ}$ \\
\hline Lam(v1) & 21 & 65 & 4 & 17 & Lam et al. (2014) & Alternative implementation of the WSLAP+ model, using a different set of strong-lensing constraints and redshifts. \\
\hline
\end{tabular}

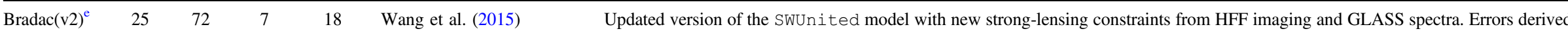
via bootstrap resampling only strong-lensing constraints.

$\begin{array}{llllll}\operatorname{Lam}(\mathrm{v} 2) & 10 & 32 & 5 & 5 & \text { Lam et al. (2014) }\end{array}$

$\begin{array}{llllll}\text { CATS(v2.1) } & 55 & 154 & 8 & 1 & \text { Jauzac et al. (2015) }\end{array}$

二 $\quad$ CATS(v2.2) $25 \quad 72 \quad 8 \quad 1 \quad$ Jauzac et al. (2015)

Updated version of the WSLAP+ model, using more selective strong-lensing constraints and

Updated version of the CATSv2 model, adopting the spec- $z$ constraints and multiple-image definitions used for Bradac(v2).

Notes.

${ }^{\text {a }}$ Number of multiply imaged systems used as strong-lensing constraints.

b Total number of multiple images used.

${ }_{\mathrm{d}}^{\mathrm{c}}$ Number of multiply imaged systems with spectroscopic redshifts.

${ }^{\mathrm{d}}$ Number of multiply imaged systems with photometric redshifts.

${ }^{\mathrm{e}}$ Uses weak lensing constraints from HST- and ground-based imaging, as described in Merten et al. (2011).

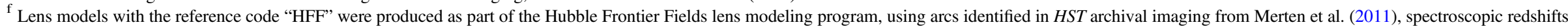

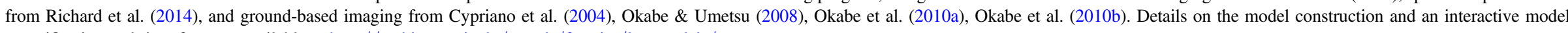
magnification web interface are available at http://archive.stsci.edu/prepds/frontier/lensmodels/.

${ }^{g}$ SWUnited: Strong and Weak lensing United; Bradač et al. (2005).

h CATS: Clusters As Telescopes lens modeling team. PI's: J.-P. Kneib \& P. Natarajan.

i LENSTOOL: Jullo et al. (2007); http://projects.lam.fr/repos/lenstool/wiki.

${ }^{\mathrm{j}}$ Uses weak lensing constraints from HST imaging, as described in Richard et al. (2014).

${ }^{\mathrm{k}}$ SaWLENS: Merten et al. (2009); strong and weak lensing analysis code. http://www.julianmerten.net/codes.html.

GRALE: Gravitational Lensing; Liesenborgs et al. (2006, 2007), Mohammed et al. (2014).

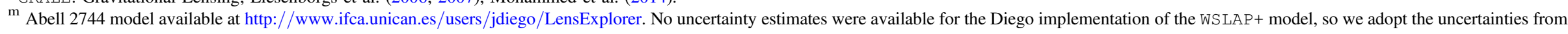
the closely related Lam model.

${ }^{n}$ WSLAP+: Sendra et al. (2014); Weak and Strong Lensing Analysis Package plus member galaxies (note: no weak-lensing constraints used for Abell 2744).

${ }^{\circ}$ GLAFIC: Oguri (2010); http://www.slac.stanford.edu/ oguri/glafic/.

p GALFIT: Two-dimensional galaxy fitting algorithm (Peng et al. 2002). 
Table 5

Lens Model Predictions for SN HFF14Tom Magnification

\begin{tabular}{lccc}
\hline \hline Model $^{\mathrm{a}}$ & Best $^{\mathrm{b}}$ & Median $^{\mathrm{c}}$ & $68 \%$ Conf. Range $^{\mathrm{d}}$ \\
\hline Bradac(v1) & 3.19 & 2.48 & $2.31-2.66$ \\
CATS(v1) & 2.28 & 2.29 & $2.25-2.34$ \\
CATS(v1.1) & $\ldots$ & 2.62 & $2.44-2.80$ \\
Merten & 2.33 & 2.24 & $2.04-2.92$ \\
Sharon(v1) & 2.56 & 2.60 & $2.44-2.78$ \\
Sharon(v2) & 2.74 & 2.59 & $2.42-2.85$ \\
Zitrin-LTM & 2.67 & 2.99 & $2.61-3.77$ \\
Zitrin-NFW & 2.09 & 2.29 & $2.07-2.52$ \\
Williams & 2.70 & 2.81 & $1.65-5.54$ \\
\hline CATS(v2) & $\ldots$ & 3.42 & $3.27-3.58$ \\
Diego & $\ldots$ & 1.80 & $1.44-2.16$ \\
GLAFIC & 2.34 & 2.29 & $2.19-2.37$ \\
Lam(v1) & $\ldots$ & 2.79 & $2.42-3.16$ \\
Bradac(v2) & 2.23 & 2.26 & $2.30-2.23$ \\
Lam(v2) & 1.86 & 1.91 & $1.54-2.28$ \\
CATS(v2.1) & $\ldots$ & 3.06 & $2.92-3.19$ \\
CATS(v2.2) & $\ldots$ & 3.07 & $2.94-3.20$ \\
\hline
\end{tabular}

Notes.

${ }^{\text {a }}$ Models above the line are from the pre-HFF set, and those below incorporate HFF data. The final four, marked with asterisks, were not formally part of the blind test as they include modifications made after the measured magnification of the SN was known.

b The magnification returned for the optimal version of each model, as independently defined by each lens modeling team.

${ }^{\mathrm{c}}$ Median magnification from 100 to 600 Monte Carlo realizations of the model.

${ }^{d}$ Confidence ranges about the median, enclosing $68 \%$ of the realized values.

Notes on the derivation of these weak-lensing constraints are given in the final column of Table 4. The signal from weak lensing relies on a large sample of background galaxies, so this constraint operates principally at separations more than $1^{\prime}$ from the cluster core. At a projected separation of $\sim 40^{\prime \prime}$, SN HFF14Tom falls in between the strong- and weak-lensing regimes.

In Figure 6 the model predictions are plotted alongside the observed magnification of SN HFF14Tom, derived in Section 6. To first order, this comparison shows that these 17 models are largely consistent with each other and with the observed magnification of SN HFF14Tom. The "naive mean" of the full set of models is $\mu_{\text {pre }}=2.6 \pm 0.4$. This is an unweighted mean (i.e., we ignore all quoted uncertainties) derived by naively treating each as an independent prediction for the magnification (this is clearly incorrect, as several models are represented multiple times as different versions). The naive mean is separated from the observed SN magnification by $\delta \mu / \mu=28 \%$, which is approximately a $1.5 \sigma$ difference.

This is approximately consistent with the results of P14 and Nordin et al. (2014), where model predictions were found to be in reasonably good agreement with a set of three lensed $\mathrm{SNe}$ from the CLASH program. The general agreement between the model predictions and the $\mathrm{SN}$ measurement is especially encouraging for these Abell 2744 models. This is a merging cluster with a complex mass distribution, and the $\mathrm{SN}$ is located outside of the strong-lensing region where the models are most tightly constrained.

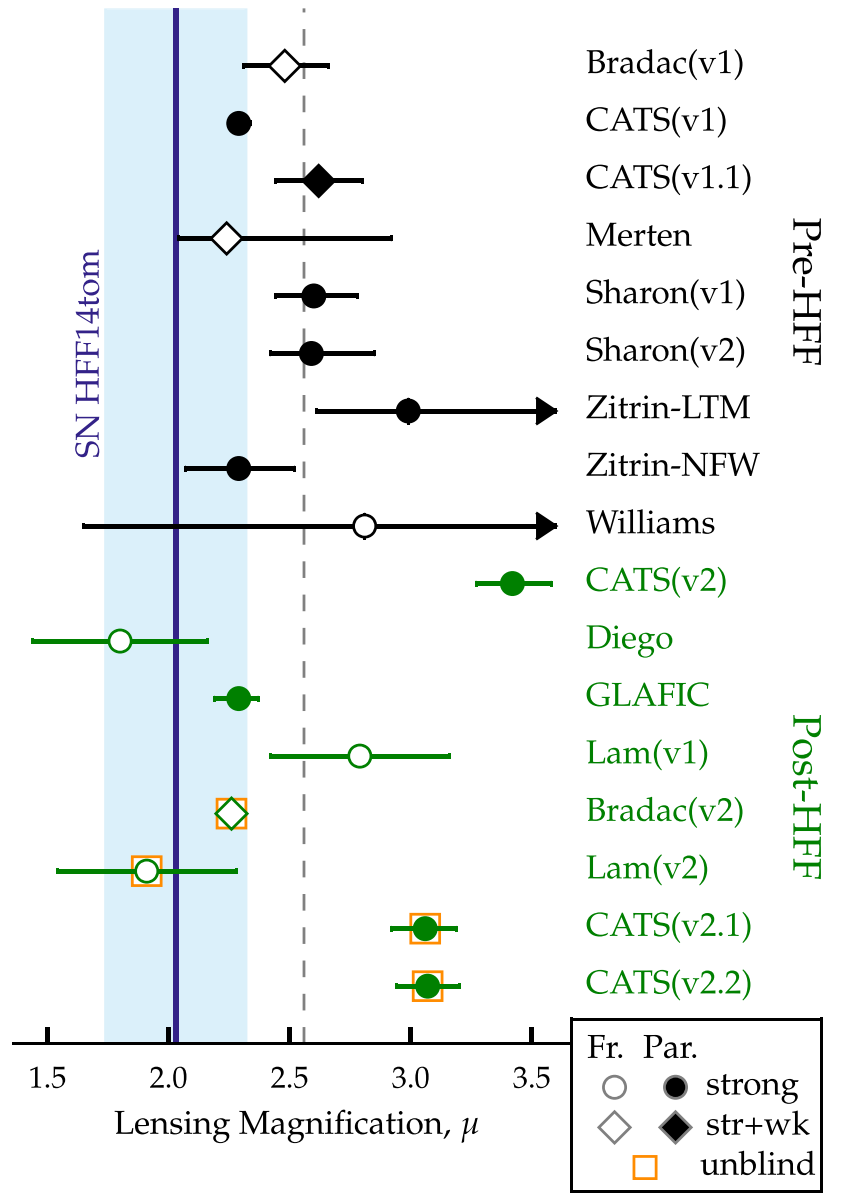

Figure 6. Comparison of the observed lensing magnification to predictions from lens models. The vertical blue line shows the constraints from SN HFF14Tom derived in Section 6 using the MLCS2k2 fitter, with a shaded region marking the total uncertainty. Markers with horizontal error bars show the median magnification and 68\% confidence region from each of the 17 lensing models. Circles indicate models that use only strong-lensing constraints, while diamonds denote those that also incorporate weak-lensing measurements. Models using a "free-form" approach are shown as open markers, while those in the "parametric" family are given filled markers. The top half, with points in black, shows the nine models that were constructed using only data available before the start of the Frontier Fields observations. The lower eight models in green used additional input constraints, including new multiply imaged systems and redshifts. The final four points, with square orange outlines, are the "unblind" models that were generated after the magnification of the SN was known. The black dashed line marks the unweighted mean for all 17 models, at $\mu=2.6$.

However, beyond this first-order agreement, there is a small systematic bias apparent. All but two of the lens models return median magnifications that are higher than the observed value, and six of the models are discrepant by more than $1.5 \sigma$. These six discrepant models are all biased to higher magnifications. They are found in both the pre-HFF and post-HFF models, in the parametric and free-form families, and among the strong-lensing-only and the strong +weak subsets. It is important to emphasize that SN HFF14Tom only samples a single line of sight through the cluster, and this bias to higher magnifications is minor. Nevertheless, a systematic shift of this nature is surprising, given the wide range of modeling strategies, input data, and physical assumptions represented by this set of models. In the following subsections we examine possible explanations for 
this small but nearly universal bias. We first consider whether a misinterpretation of the data on the SN itself can account for the observed systematic bias, and then examine the lens models.

\subsection{Possible Errors in SN Analysis}

\subsubsection{Redshift Error}

If the redshift of the SN derived in Section 4 were incorrect, then one would derive a different value for the magnification, both from the SN measurement and the lens model predictions. Conceivably, this could resolve the tension between the measurement and the models. It is often the case in SN surveys that redshifts are assigned based on a host galaxy association, typically inferred from the projected separation between the $\mathrm{SN}$ and nearby galaxies. In this case the redshift is strongly supported by evidence from the SN itself: we find a consistent redshift from both the SN spectrum (Section 4.2) and the light curve Section 5, which are both within $1 \sigma$ of the spectroscopic redshift for the nearest detected galaxy: $z=1.3457$. This appears to be a solid and self-consistent picture, so the evidence strongly disfavors any redshift that is significantly different from $z=1.35$.

We have adopted the most precise redshift of $z=1.3457$ from the host galaxy as our baseline for the magnification comparison. If instead we adopt the spectroscopic redshift from the SN itself $(z=1.31$; Section 4.2) then we find no significant change in the inferred magnifications or in the suggestion of a small systematic bias.

\subsubsection{Foreground Dust and SN Color}

All SN sight-lines must intersect some amount of foreground dust from the immediate circumstellar environment, the host galaxy, and the intergalactic medium. In the case of SN HFF14Tom one might posit some dust extinction from the intracluster medium (ICM) of Abell 2744, although measurements of rich clusters suggest that the ICM has only a negligible dust content (Maoz 1995; Stickel et al. 2002; Bai et al. 2007). When fitting the HFF14Tom light curve we account for dust by including corrections that modify the inferred luminosity distance based on the SN color. If after applying these dust corrections we were still underestimating the effect of dust along this sight-line, then the SN would appear dimmer than it really is, the inferred distance modulus would be higher, and the measured magnification would be biased to an artifically low value. Thus, an underestimation of dust would be in the right direction to match discrepancy we observe.

In Section 6.1 we found that SN HFF14Tom is on the blue end of the normal range of Type Ia SN colors. With the SALT2 fitter we measured a color parameter $c=-0.127 \pm 0.025$, and with MLCS2k2 we found the host galaxy dust extinction to be $A_{V}=0.011 \pm 0.025 \mathrm{mag}$. These colors are tightly constrained, as we are fitting to photometry that covers a restframe wavelength range from $\sim 3500$ to $7000 \AA$ and extends to $\sim 30$ days past maximum brightness. This leaves little room for the luminosity measurement to be biased by dust, as the dimming of HFF14Tom would also necessarily be accompanied by some degree of reddening.

Nevertheless, one might suppose that a bias could be introduced if we have adopted incorrect values for the color correction parameter $\beta$ or extinction law $R_{V}$ in the SALT2 and
MLCS2k2 fits, respectively. The appropriate value to use for this color correction and how it affects inferences about the intrinsic scatter in Type Ia SN luminosities is a complex question that is beyond the scope of this work (see, e.g., Chotard et al. 2011; Marriner et al. 2011; Kessler et al. 2013; Scolnic et al. 2014b). However, we can already rule this out as a solution for the magnification discrepancy. Our error on the HFF14Tom distance modulus already includes an uncertainty in the extinction law and a related error to account for the intrinsic luminosity scatter. These are well vetted parameters, based on observations of $\sim 500 \mathrm{SNe}$ extending to $z \sim 1.5$ (Sullivan et al. 2011). Furthermore, there is no reason to propose that SN HFF14Tom is uniquely affected by a peculiar type of dust. Thus, any change in the color correction applied to SN HFF14Tom would require the same adjustment to be applied to the unlensed SNe at similar redshift that make up our comparison sample, largely negating the effect on the inferred magnification.

The traditional color corrections as formalized in SN light curve fitters are designed to account for a dust component that lies in the rest frame of the SN. The inferred luminosity of a SN can also be affected by the presence of foreground dust with a different redshift and possibly a different reddening law (Ménard et al. 2010a). However, the magnitude of such a bias is insufficient to account for the observed discrepancy, Ménard et al. (2010b) estimate the opacity of the universe as $\left\langle A_{V}\right\rangle \sim 0.03 \mathrm{mag}$ up to $z=0.5$. While this can have a measurable impact on precise cosmological constraints, it is far less than the $0.23 \mathrm{mag}$ difference between the observed magnification of HFF14Tom and the mean of the model predictions.

Although the very blue color of SN HFF14Tom is helpful to rule out dust as a possible explanation for the observed small systematic bias, it is possible that this very blue color is itself leading to a bias in the distance measurement. Scolnic et al. (2014a) measured a small bias in the SALT2 fitter for Type Ia $\mathrm{SNe}$ that have a color parameter derived from the light curve fit $c<-0.1$ (see, e.g., their Figure 10 and Section 5.1). For HFF14Tom we have measured $c=-0.13$, which would correspond to a bias of roughly $+0.05 \mathrm{mag}$ in the SALT2 distance measurement. If we were to apply a $-0.05 \mathrm{mag}$ correction to the distance modulus from the SALT2 fit, then this would increase the inferred magnification to $\mu_{\text {SALT2 }}=2.08 \pm 0.36$. This would slightly reduce the tension between the SALT2 measurement and the model predictions, though it would not be enough to completely alleviate it.

\subsubsection{Misclassification}

Is it possible that HFF14Tom is an example of a peculiar stellar explosion that does not follow the relationship between light curve shape and luminosity observed in normal Type Ia SNe? In Sections 4.1 and 5 we classified SN HFF14Tom as a normal Type Ia SN based on both the spectroscopic and photometric evidence. This rules out the possibility that HFF14Tom belongs to a different class of normal SN explosions (Type Ib, Ic, or II). In Section 6.1 we fit the HFF14Tom light curve to determine a luminosity distance and found that the light curve shape and color are consistent with a Type Ia SN of average light curve width, with minimal dust extinction. This excludes the possibility that HFF14Tom is 


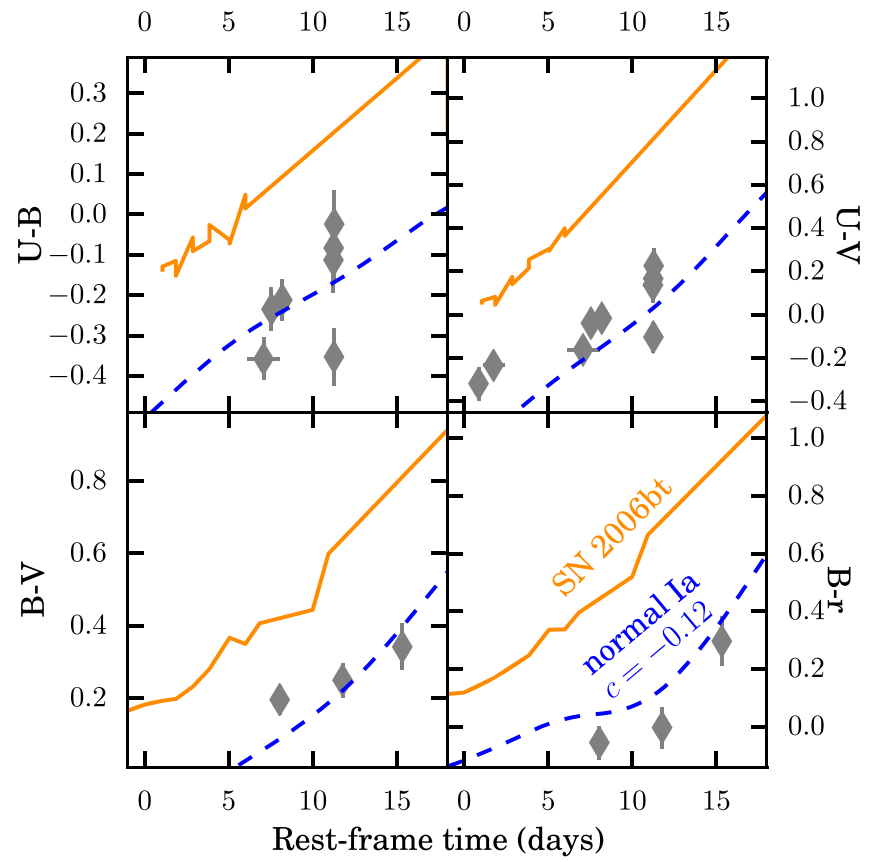

Figure 7. Comparing HFF14Tom observations to the color curves of SN 2006bt and a normal Type Ia SN model. Gray points show the observed colors of SN HFF14Tom, $k$-corrected to rest-frame $U B V r$ bands. Solid orange lines show the observed color curves for the peculiar Type Ia SN 2006bt. Dashed blue lines show the color curves for a normal Type Ia SN, derived from the SALT2 model with a color parameter $c=-0.13$, set to match the best-fit color for SN HFF14Tom.

one of the sub-class of faint and fast-declining Type Ia SNe like SN 1991bg.

One remaining possibility is that HFF14Tom could be part of a rare sub-category of peculiar Type Ia SNe that masquerade as their normal cousins, epitomized by the prototype SN 2006bt (Foley et al. 2010). These objects appear to have a normal Type Ia light curve shape, except for the absence of a secondary maximum or "shoulder" in near-IR bands. The reddest filter available for the HFF14Tom light curve is F160W, which has an effective rest-frame wavelength of $6623 \AA$ at $z=1.31$, making it close to the rest-frame $r$ band. Although the near-IR shoulder is more prominent in the restframe $i$ band, we can see in Figure 4 that the F160W light curve may suggest a weak or delayed near-IR shoulder. The secondto-last observation in $\mathrm{F} 160 \mathrm{~W}$ is $\sim 1 \sigma$ lower than predicted by the best-fit SALT2 and MLCS2k2 models. This is tenuous evidence, but it would be consistent with a 2006bt-like light curve.

In this case, however, the color of SN HFF14Tom can rule out a classification as a 2006bt-like object. All members of this peculiar sub-class exhibit very red colors across all optical and near-IR bands, consistent with a very cool photosphere (Foley et al. 2010). Figure 7 shows that HFF14Tom is bluer than the SN 2006bt prototype by at least 0.25 mag in every color. The observed HFF14Tom colors are fully consistent with a normal, blue Type Ia SN (the SALT2 model is shown).

\subsection{Possible Errors in Lens Modeling}

Having found no evidence for misinterpetations of the SN data, we are left to seek an explanation for the mild tension by scrutinizing the lens models. Here we evaluate four possible ways in which the input data or assumptions of these lens models could lead to a systematic bias in the magnification.

\subsubsection{Unconstrained Mass Model Extrapolation}

As noted above, the position of SN HFF14Tom just beyond the edge of the strong-lensing region means that all models relying only on strong-lensing constraints must extrapolate the mass profile in order to make a prediction for the HFF14Tom magnification. Let us suppose that the true cluster density profile for Abell 2744 happens to have a sharp drop right at the edge of this core strong-lensing region. In that case these strong-lensing only models might well overestimate the mass interior to the HFF14Tom position, and thus systematically overestimate the magnification.

One might expect that models incorporating weak-lensing constraints would be less susceptible to such bias from a sharply steepening mass profile. However, we find that collectively the four strong+weak lensing models (shown as diamonds in Figure 6) exhibit the same propensity to overestimate the magnification along this line of sight. This comparison therefore provides no evidence to support the extrapolation error hypothesis.

With two versions of the CATS(v1) model, we also have a more direct test of the effect of introducing weak lensing constraints. The initial CATS(v1) model uses only stronglensing constraints, and gives $\mu=2.29_{-0.04}^{+0.05}$, slightly higher than the measured value. A second iteration of this model, labeled here as CATS(v1.1), used the same strong-lensing features, but added in weak lensing constraints. The revised magnification of $\mu=2.62 \pm 0.18$ is further from the measured value, which serves to reinforce the conclusion that adding weak lensing constraints cannot, by itself, resolve this bias.

Given the intermediate location of this $\mathrm{SN}$, caught between the strong-lensing and weak-lensing regimes, an even more useful tool than weak lensing would be the flexion signal (Goldberg \& Natarajan 2002; Goldberg \& Bacon 2005; Irwin \& Shmakova 2006; Goldberg \& Leonard 2007). Flexion is a second-order lensing effect that distorts the shapes of background galaxies that are not lensed strongly enough to be warped into arcs or filaments. A flexion measurement near the SN HFF14Tom sight line would be more sensitive to local gradients and substructure in the cluster mass profile. However, no such flexion constraints are yet available.

\subsubsection{Line of Sight Structure}

In addition to the dominant gravitational lensing from the foreground cluster, a distant source like SN HFF14Tom will also be subject to the cumulative weak lensing effects due to uncorrelated large scale structure (LSS) along the line of sight, sometimes called "Cosmic Weak Lensing" (Wong et al. 2011; Host 2012; Collett et al. 2013; Greene et al. 2013; Bayliss et al. 2014; D'Aloisio et al. 2014; McCully et al. 2014). This LSS effect is most important for very high redshift sources $(z>5)$, which have a much longer path length over which to encounter LSS and for highly magnified sources very near to the cluster lensing critical curve (because LSS can perturb the position of the critical curve). For SN HFF14Tom at the modest redshift of $z=1.35$ and far from the critical curve, LSS should increase the scatter in the lensing magnification at a level much less than $10 \%$ (D'Aloisio et al. 2014). The LSS effect is therefore unable to account for the observed discrepancy of $\sim 23 \%$ by itself. 
In addition, some sources of uncertainty are taken into account only by a subset of models. For example, the Willams model (using GRALE; Liesenborgs et al. 2006, 2007; Mohammed et al. 2014) accounts for mass sheet degeneracy by introducing a free parameter in the reconstruction that represents an arbitrarily scaled mass sheet. The flexibility of the Williams model and the inclusion of this source of uncertainty contribute to its relatively large uncertainties compared to the other models.

\subsubsection{Nearby Cluster Member}

A component of the Abell 2744 lens that is particularly relevant to SN HFF14Tom is the cluster member galaxy that lies just 5!. 8 north of the SN position (see Figure 1). If the mass-to-light ratio $(M / L)$ or the profile of the dark matter halo for this galaxy were significantly different from other cluster member galaxies, then its proximity to the SN sight-line might drive a bias in the magnification. We tested this hypothesis using the CATS(v2) model by allowing the mass of the nearby cluster member galaxy to vary as a free parameter in the model. We found that the change in the SN HFF14Tom magnification prediction was less than $\Delta \mu=0.1$. This additional dispersion is already included in the uncertainties quoted for that model in Table 5 and Figure 6. Furthermore, an erroneous $M / L$ value for the nearby cluster member galaxy could not explain the systematic shift of all lens models, because some do not incorporate cluster member galaxies into their constraints at all (the free-form Bradac, Williams, and Merten models).

\subsubsection{Cosmological Parameter Uncertainty}

Most lens models make a fixed assumption for the values of cosmological parameters in a standard $\Lambda \mathrm{CDM}$ cosmology (typically: $\Omega_{\mathrm{m}}=0.3, \Omega_{\Lambda}=0.7, H_{0}=70 \mathrm{~km} \mathrm{~s}^{-1} \mathrm{Mpc}^{-1}$ ). This can introduce a systematic magnification error that is comparable in magnitude to that of the statistical uncertainties (Zitrin et al. 2014; Bayliss et al. 2015). Incorporating this cosmological parameter uncertainty would increase the model magnification errors, and therefore reduce the tension between models and observation, but would not resolve the overall systematic shift. Bayliss et al. (2015) find that for Abell 2744 the additional cosmological uncertainty for magnifications $\mu \approx 2$ is $<10 \%$ which would still leave six models discrepant by at least $1.5 \sigma$.

\subsubsection{Misidentification of a multiple image}

Jauzac et al. (2015) proposed a correction for the location of image 3.3, the third component of a triply imaged galaxy (Merten et al. 2011) at $z=3.98 \pm 0.02$ (Johnson et al. 2014). This possibly specious multiple image is only $\sim 10^{\prime \prime}$ from the SN HFF14Tom position, meaning a change in its location could have a large impact on the predicted magnification for SN HFF14Tom (see Johnson et al. 2014, for a quantitative discussion of this effect). However, Jauzac et al. (2015) also evaluated a version of the CATS(v2) model in which this single source is left in the original position and found that the location of image 3.3 does not significantly affect the predicted magnifications. Furthermore, dividing our 17 models into those using the original location versus those that adopt the new position, we find that both groups include models that are within $1 \sigma$ of the observed $\mu$ as well as significantly discrepant points.

\subsubsection{Source Plane Minimization}

A general tendency toward high model magnification can result when lens models are minimized in the source plane (rather than the image plane). For a given model, modifying the surface mass density to have a shallower profile (declining less quickly with radius) will lead to an increase in the predicted magnifications across the field. As the magnification applies to both flux and area, the shallower profile also leads to a smaller source plane area, which necessarily brings delensed images of the same source closer together in the source plane. Thus, models that are naively optimized in the source plane will have a tendency toward shallower mass profiles and larger magnifications, which force all sources toward the same location in the source plane (see, e.g., Kochanek 1991, 2006; Jee et al. 2007; Ponente \& Diego 2011).

There are several strategies for mitigating this well known bias. Optimizing a model in the image plane can avoid this bias entirely (Broadhurst et al. 2005; Zitrin et al. 2009). The Sharon (v2), Zitrin-LTM, Zitrin-NFW, and CATS(v1, v2-2.2) models all use image plane minimization. Alternatively, one may guard against this bias by using a modified source plane minimization that is constrained not to generate overly small de-lensed sources (Diego et al. 2014; Sendra et al. 2014). In our test set, a version of this second strategy is employed by the GLAFIC, Williams, and Diego models. For example, the Williams model uses source plane optimization in the GRALE algorithm, but avoids bias by maximizing the fractional overlap of extended source plane images. Finally, the effect of this bias should be reduced when one uses many strong lensing constraints over a wide range of redshifts. The CATS(v2) and CATS(v2.1) models have the largest number of strong-lensing constraints, using 50 and 55 multiply imaged systems, respectively.

Although most of the tested models utilize one of these strategies to prevent a source plane minimization bias, Table 5 and Figure 6 show that these models do not uniformly deliver more accurate magnification predictions. The most direct test of this is in the Sharon and CATS model series. Both of these model series use the LENSTOOL software (Kneib et al. 1996; Jullo et al. 2007; Jullo \& Kneib 2009; Kneib et al. 2011), which allows a choice of how the minimization is done. For the Sharon series, the v1 model used the faster source plane minimization approach, but the v2 model was constructed with the more accurate image plane minimization. The CATS v1, 2, 2.1, and 2.2 models all used image plane optimization, while the v1.1 employed source plane optimization (and also added in weak lensing constraints). In neither of these model series do we see a uniform improvement in accuracy when optimization is done in the image plane. This test strongly suggests that the observed tension between the lens models and the SN observation cannot be entirely attributed to a bias arising from source plane minimization.

\subsection{Quantity and Quality of Strong Lensing Constraints}

None of the possible errors in lens model inputs described above can completely account for the mild tension between the observed and predicted magnifications. However, we do have several models that agree with the measured SN magnification. In this section we take a simple pragmatic approach and seek to identify any shared characteristics of the models that are most accurate for this particular sight-line. In this way SN HFF14Tom may serve as a guide toward optimizing future 


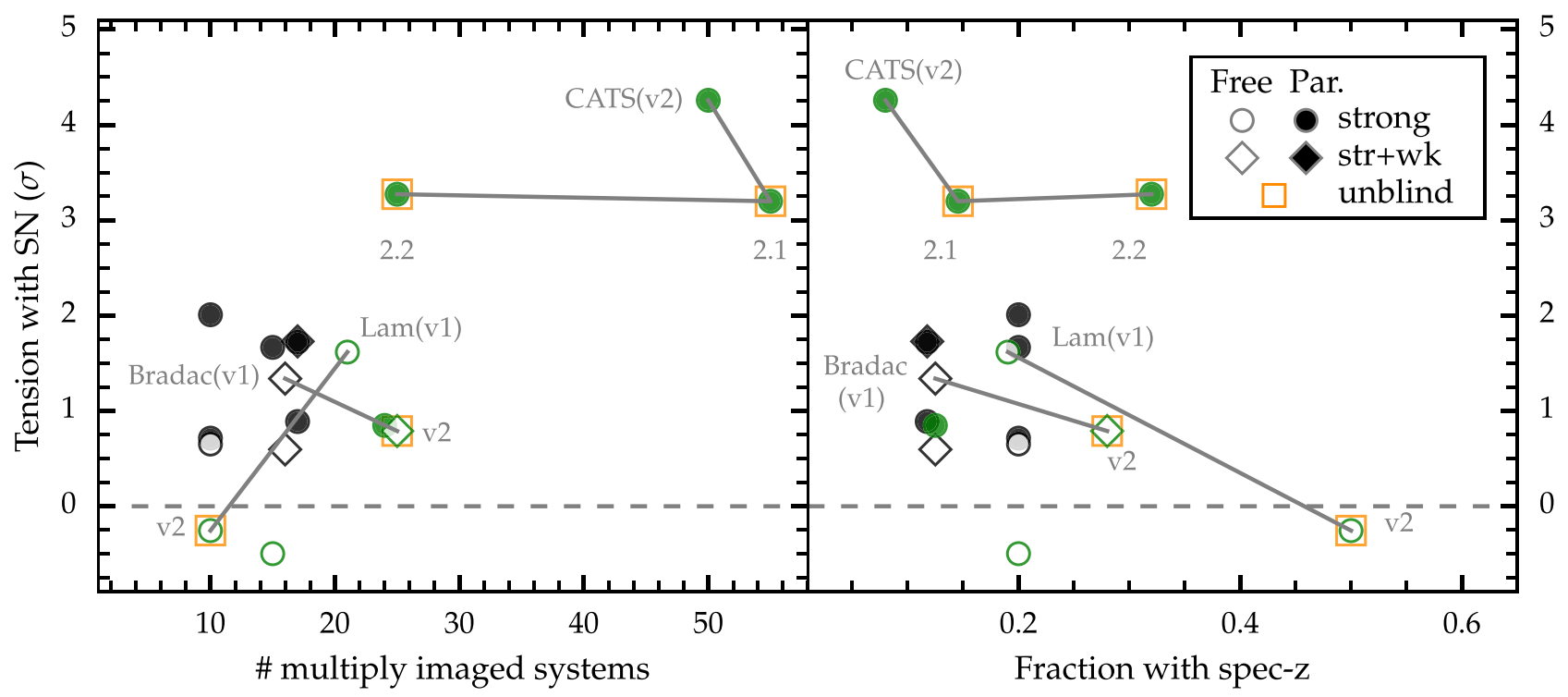

Figure 8. Model tension as a function of strong-lensing constraints. The $y$ axis in both panels marks the number of standard deviations in discrepancy between the measured SN magnification and the prediction from each lens model: $\left(\mu_{\text {model }}-\mu_{\mathrm{SN}}\right) /\left(\sigma_{\text {model }}^{2}+\sigma_{\mathrm{SN}}^{2}\right)^{1 / 2}$. The left panel plots this tension against the total number of multiply imaged systems used, and the right panel plots the fraction of multiply imaged systems that have a spectroscopic redshift constraint. Marker shapes and colors are the same as in Figure 6. In both panels, three lens model sequences are highlighted with connecting lines: the Bradac, Lam, and CATS(v2) models, which are the three examples where the number of strong lensing constraints and the fraction with spectroscopic redshifts changed substantially from one model version to the next.

lens models for magnification predictions, particularly in regions where strong-lensing constratins may be scarce.

The addition of deeper imaging and new spectroscopic constraints from the HFF program and affiliated efforts might be expected to improve the accuracy of magnification predictions. However, in Figure 6 we saw no evidence for a universal improvement in model accuracy when moving from the pre- to the post-HFF models. In Figure 8 we attempt to refine this hypothesis by isolating the quantity and quality of strong lensing constraints.

The left panel plots the tension between each model prediction and the measured SN magnification against the number of multiply imaged galaxies used as strong-lensing constraints. The Bradac, Lam, and CATSv2 model series are highlighted here with connecting lines, as three examples where the number of systems used has changed substantially from one version to the next, but the model construction has remained fundamentally unchanged. There is no clear correlation here, and in fact for both the Lam and CATSv2 model series, we see the tension actually increases for a larger number of strong-lensing constraints.

In the right panel of Figure 8 we examine an alternative explanation by plotting the model tension against the fraction of strong-lensing constraints that have a spectroscopic redshift. For the SN HFF14Tom magnification prediction, we see that all three of the testable model series (Bradac, Lam, CATSv2) become more accurate as the spec- $z$ fraction increases. ${ }^{47}$ The spec- $z$ fraction here serves as a crude stand-in for measuring how stringently the strong-lensing constraints are selected in these models.

The SN HFF14Tom comparison therefore supports the notion that the quality of strong-lensing constraints is just as important as the quantity —at least for computing magnifications of sources outside the strong-lensing region. Once a

\footnotetext{
47 Of course this apparent correlation does not imply any direct causation Consider the limit of just a single multiply imaged galaxy constraint: the spec- $z$ fraction could be 1.0 but the model would be wildly unconstrained.
}

sufficient number of robust multiply imaged systems have been included (perhaps $\sim 10$ ) most models will be dominated by systematic uncertainties such as those described in Section 7.2. To improve the accuracy of magnification predictions with a large number of strong-lensing constraints, one should employ a strict vetting of the multiply imaged systems. Each system used should ideally have a spectroscopic redshift, or at least a very well constrained photometric redshift. The primary value in this strict vetting is to avoid erroneous redshifts being assigned to multiply imaged systems that are unconstrained in redshift space. Such redshift errors are especially problematic for relatively low $z$ background systems: at lower redshifts the model-derived deflection angle and magnification show a more rapid variation with the redshift of the background galaxy than for similarly positioned high redshift galaxies. This conclusion should be unsurprising, as for example, Bayliss et al. (2015) have shown that cosmological uncertainties are smaller for models that use a large fraction of strong-lensing constraints with spectroscopic redshifts, and Johnson et al. (2014) have shown a case study where adding spectroscopic redshift information changes the inferred magnification by $\sim 10 \%$.

It is relevant to note that the three model series highlighted here (Bradac, CATSv2, and Lam) also happen to be the three models that are not truly "blind," in that the modelers were aware of preliminary analysis of the SN magnification when constructing the latest version. However, none of these models used the SN magnification as an input constraint, and none were consciously tuned to match the SN magnification. In fact, for the Bradac(v2) and Lam(v2) cases, the modelers did not have access to a final measurement of the SN magnification at the time their models were generated.

\section{SUMMARY AND CONCLUSIONS}

The appearance of a Type Ia SN behind a massive galaxy cluster provides a rare opportunity to use a standard candle for a direct measurement of the absolute magnification due to gravitational lensing. The discovery of SN HFF14Tom in the 
HFF imaging of Abell 2744 offers the first chance to apply this test on a cluster with multiple publicly available lens models. We have found that the spectrum and light curve of SN HFF14Tom are well matched by templates of a normal Type Ia SN at $z=1.3457$. Using the two most prevalent Type Ia SN light curve fitters, SALT2 and MLCS2k2, we get a consistent measurement of the distance modulus (Table 3). Using a cosmology-independent comparison against a sample of unlensed Type Ia SNe at similar redshifts, we find that SN HFF14Tom is $\sim 0.7$ magnitudes brighter than the field sample would predict. Attributing this difference to the gravitational lensing magnification (and accounting for the intrinsic scatter in luminosity of the Type Ia $\mathrm{SN}$ population), we have derived a consistent measured magnification of $\mu_{\text {SALT } 2}=1.99 \pm 0.38 . \quad \mu_{\text {MLCS2k2 }}=2.03 \pm 0.29$, from the two light curve fitters.

Taking advantage of the availability of 17 well-constrained lens models for the Abell 2744 cluster, we have used SN HFF14Tom to ask how accurately these lens models can predict the magnification along this line of sight. We find that these models are consistent, and fairly accurate, collectively predicting $\mu=2.5 \pm 0.4$, within $1 \sigma$ of the measured value. This is encouraging, and reinforces the quality and value of these public lens models for studying magnified background objects. However, we note that all but two of the tested models provide a median magnification prediction that is larger than the measurement from the SN, and all the models with significant discrepancy are too high. This may be an indication that there is a small systematic bias at work, at least in some of the models.

We have speculated on what could be the origin for such a systematic bias, first considering and rejecting three possible explanations that presume an error in the interpretation of the available SN data.

1. Redshift: We reject the possibility that a redshift error is the primary cause of the discrepancy, as the redshift evidence is well supported by multiple lines of evidence from both the $\mathrm{SN}$ and the presumed host galaxy. Changing the redshift within the constraints of these complementary observations does not resolve the tension.

2. Dust: We find it implausible that there is sufficient dust in the cluster or elsewhere along the line of sight to account for the magnification discrepancy. A dust-induced bias is also disfavored by the very blue color of SN HFF14Tom.

3. Misclassification: The combination of spectroscopic and photometric evidence strongly supports our classification of SN HFF14Tom as a normal Type Ia SN. The most plausible mis-classification would be that the object is a peculiar Type Ia of the SN 2006bt-like sub-class. Although the light curve shape could allow this possibility, the blue color of HFF14Tom can once again reject this alternative.

Turning to the lens models, we have considered six ways in which a bias might be introduced into the lens models:

1. Mass profile extrapolation errors.

2. Large scale structure along the line of sight.

3. Peculiar mass for a nearby cluster member.

4. Cosmological parameter uncertainty.

5. Misidentification of a multiple image.

6. Source plane minimization.
Although each of these could plausibly introduce a small systematic bias, we find that none are likely to be significant enough (or universal enough) to completely resolve the tension between models and observations.

Finally, we have examined whether there is a simple prescription for the kind of strong lensing constraints that are most likely to deliver an accurate magnification prediction for this sight-line. We find that the number of multiply imaged systems used is not in itself predictive of model accuracy. Rather, it is the fraction of multiply imaged systems that have spectroscopic redshifts that is most correlated with model accuracy. A reasonable interpretation-at least for this cluster and this particular set of models-is that the spectroscopic redshift fraction serves as an effective proxy for the "quality" of the strong lensing constraints. This quality of the input data appears to be a key ingredient for deriving accurate magnifications outside the strong-lensing regime.

We have evaluated here only a single object behind a single cluster, with a minor tension between the observed magnification and the model predictions. This is not in and of itself a cause for alarm. Previous analyses of lensed Type Ia SN found no significant discrepancy between the observed Type Ia SN magnifications and the predictions from lens models (Nordin et al. 2014; P14), albeit with a much smaller set of lens models being tested. The observed systematic bias for HFF14Tom is small, and many of the lens models that deviate from the measured magnification are preliminary models that have not been updated to include all of the HFF data. Future revisions of the lens models for Abell 2744 could either incorporate the observed magnification of HFF14Tom as a new model constraint, or can revisit this test to evaluate whether the bias persists.

The general problem of combining predictions from many independent models has a rich history in the astronomical and statistical literature (e.g., Press 1997; Hoeting et al. 1999; Liddle 2009). In a recent example, Dahlen et al. (2013) examined the photometric redshifts from multiple galaxy SED fitting codes using the same input data. They found that the photo- $z$ accuracy and precision can be substantially improved by combining the outputs using a sum of probability distributions, a hierarchical Bayesian method, or even a technique as simple as taking the straight median from all available models. It may be that lensing magnifications are similar, in that they can in general be improved through a simple combination of lens model predictions. Such model averaging will not, however, resolve a systematic bias that is shared by many models.

\subsection{Future Work}

A promising avenue for exploring the origins of such systematic biases in cluster lens models is through the use of simulated lensing data. One can start with very deep highresolution multi-band imaging on an unlensed field that has a fairly complete spectroscopic redshift catalog, such as the Hubble Ultra Deep Field. Then a simulated galaxy cluster is placed in the field, and the background galaxies are distorted into arcs and multiple images using a well-defined lensing prescription. The artificially lensed images can then be distributed to lens modeling teams who attempt to reconstruct the (known) mass profile of the simulated cluster. This exercise has recently been pursued with a set of synthetic clusters similar to those observed in the $\mathrm{HFF}$ program 
(M. Meneghetti et al. 2015, in preparation), using techniques similar to Meneghetti et al. (2010, 2014). Preliminary analysis of this simulation comparison suggests that magnifications can be systematically overestimated for sources that lie outside the strong-lensing region along the minor axis direction of a simulated cluster that is very elongated, similar to Abell 2744. This indicates that some lens models significantly underestimate the ellipticity of the mass distribution on large scales for that simulated cluster, which is consistent with a recent lens model comparison using 25 real clusters from the CLASH program (Zitrin et al. 2015). Our analysis of SN HFF14Tom here indicates that such simulation efforts and head-to-head lens model comparisons will be an important step for moving toward precision science with cluster-lensed sources.

Increasing the sample of $\mathrm{SNe}$ behind clusters like those in the HFF program-with rich lensing constraints and deep imaging-would allow the test described here to be repeated and refined. With 10 or 100 such objects, it would be possible to see whether the SN HFF14Tom $\mu$ discrepancy is simply an outlier, or an indication of a more pernicious systematic error. Any cluster that has been vetted by pencil-beam magnification tests using lensed Type Ia SN will be able to provide a more reliable measure of the magnifications for very high redshift objects. Similarly, a broad sample of SNe like HFF14Tom would help to define the preferred lens modeling methodology by highlighting any models that consistently perform well in Type Ia SN lensing tests. The ongoing FrontierSN program will discover and follow any more highly magnified $\mathrm{SNe}$ that appear behind the Frontier Field clusters. Unfortunately, the HFF survey is not designed with high- $z$ transient discovery as a primary science goal, so the FrontierSN effort will likely add no more than 1-3 new lensed Type Ia SN. Further imaging of strong-lensing clusters with HST or the James Webb Space Telescope could enable a larger sample to be collected, especially if the filters and cadence are optimized for detection of Type Ia SN at $z>1$. Massive clusters such as Abell 2744 will continue to be attractive as cosmic telescopes, allowing the next generation of telescopes to reach the faintest objects in the very early universe. The puzzling bias revealed by SN HFF14Tom supports a concerted effort to improve these lenses with further examination of lensing systematics through simulations and collection of a larger sample of magnified SNe.

This work is dedicated to our colleague and friend Tomas Dahlen, for whom this supernova has been named in memoriam. He is dearly missed.

We thank the Hubble Frontier Fields team at STScI for their substantial efforts to make the HFF program successful. In particular, thanks are due to Matt Mountain for the allocation of discretionary orbits for the HFF program; to Jennifer Lotz, Norman Grogin, and Patricia Royle for accommodations in strategy and implementation to make the FrontierSN program possible; to Anton Koekemoer for $\mathrm{HFF}$ data processing support. We also thank the CLASH team, led by Marc Postman, for observations, catalogs, and high level science products that were of significant value for this analysis. Thanks to Jonatan Selsing for helpful comments on the manuscript.

This work utilizes gravitational lensing models produced by modeling teams that were funded as part of the HST Frontier Fields program conducted by STScI. STScI is operated by the Association of Universities for Research in Astronomy, Inc. under NASA contract NAS 5-26555. The lens models were obtained from the Mikulski Archive for Space Telescopes (MAST).

Financial support for this work was provided to S.A.R. by NASA through grants HST-HF-51312 and HST-GO-13386 from STScI, which is operated by Associated Universities for Research in Astronomy, Inc. (AURA), under NASA contract NAS 5-26555. A.M. acknowledge the financial support of the Brazilian funding agency FAPESP (Post-doc fellowshipprocess number 2014/11806-9). Support for this research at Rutgers University was provided in part by NSF CAREER award AST-0847157 to SWJ. The Dark Cosmology Centre is supported by the Danish National Research Foundation. J.M.D. acknowledges support of the consolider project CSD2010-00064 and AYA2012-39475-C02-01 funded by the Ministerio de Economia y Competitividad. J.M. contributed to this research from the Jet Propulsion Laboratory, California Institute of Technology, under a contract with NASA and acknowledges support from NASA Grants HST-GO-13343.05-A and HSTGO-13386.13-A. The research leading to these results has received funding from the People Programme (Marie Curie Actions) of the European Union's Seventh Framework Programme (FP7/20072013) under REA grant agreement number 627288. A.Z. acknowledges financial support from NASA through grant HST-HF-51334.01-A awarded by STScI and operated by AURA. T.T. acknowledges support by the Packard Foundation in the form of Packard Research Fellowship. GLASS is funded by NASA through HST grant GO-13459. L.L.R.W. acknowledges the support of the Minnesota Supercomputing Institute.

Facility: HST (WFC3).

\section{REFERENCES}

Alavi, A., et al. 2014, ApJ, 780, 143

Amanullah, R., et al. 2015, arXiv:1504.02101

Bai, L., Rieke, G. H., \& Rieke, M. J. 2007, ApJL, 668, L5

Bartelmann, M. 2010, CQGra, 27, 233001

Bayliss, M. B., Sharon, K., \& Johnson, T. 2015, ApJL, 802, L9

Bayliss, M. B., et al. 2014, ApJ, 783, 41

Benítez, N. 2000, ApJ, 536, 571

Betoule, M., et al. 2014, A\&A, 568, A22

Blondin, S., \& Tonry, J. L. 2007, ApJ, 666, 1024

Bouwens, R. J., et al. 2009, ApJ, 690, 1764

Bouwens, R. J., et al. 2014, ApJ, 795, 126

Bradač, M., Lombardi, M., \& Schneider, P. 2004, A\&A, 424, 13

Bradač, M., et al. 2005, A\&A, 437, 39

Bradač, M., et al. 2009, ApJ, 706, 1201

Brammer, G. B., et al. 2012, ApJS, 200, 13

Broadhurst, T., et al. 2005, ApJ, 621, 53

Childress, M. J., Wolf, C., \& Zahid, H. J. 2014, MNRAS, 445, 1898

Chotard, N., et al. 2011, A\&A, 529, L4

Coe, D., \& Moustakas, L. A. 2009, ApJ, 706, 45

Coe, D., et al. 2013, ApJ, 762, 32

Collett, T. E., et al. 2013, MNRAS, 432, 679

Conley, A., et al. 2008, ApJ, 681, 482

Conley, A., et al. 2011, ApJS, 192, 1

Cypriano, E. S., et al. 2004, ApJ, 613, 95

Dahlen, T., et al. 2013, ApJ, 775, 93

D’Aloisio, A., Natarajan, P., \& Shapiro, P. R. 2014, MNRAS, 445, 3581

Diego, J. M. 2014, arXiv:1406.3036

Diego, J. M., et al. 2014, arXiv:1402.4170

Diego, J. M., et al. 2015, MNRAS, 447, 3130

Dobke, B. M., et al. 2009, MNRAS, 397, 311

Ellis, R., et al. 2001, ApJL, 560, L119

Falco, E. E., Gorenstein, M. V., \& Shapiro, I. I. 1985, ApJL, 289, L1

Filippenko, A. V. 1997, ARA\&A, 35, 309

Foley, R. J., Filippenko, A. V., \& Jha, S. W. 2008, ApJ, 686, 117

Foley, R. J., et al. 2010, ApJ, 708, 1748

Foley, R. J., et al. 2012, ApJL, 753, L5 
Foley, R. J., et al. 2014, MNRAS, 443, 2887

Franx, M., et al. 1997, ApJL, 486, L75

Fruchter, A. S., et al. 2010, in STSCI Calibration Workshop Proc., ed. S. D. C. Oliveira, 376

Goldberg, D. M., \& Bacon, D. J. 2005, ApJ, 619, 741

Goldberg, D. M., \& Leonard, A. 2007, ApJ, 660, 1003

Goldberg, D. M., \& Natarajan, P. 2002, ApJ, 564, 65

Goobar, A., et al. 2009, A\&A, 507, 71

Graur, O., et al. 2014, ApJ, 783, 28

Greene, Z. S., et al. 2013, ApJ, 768, 39

Gunnarsson, C., \& Goobar, A. 2003, A\&A, 405, 859

Guy, J., et al. 2010, A\&A, 523, A7

Hoeting, J. A., et al. 1999, StaSc, 14, 382

Holtzman, J. A., et al. 2008, AJ, 136, 2306

Holz, D. E. 2001, ApJL, 556, L71

Host, O. 2012, MNRAS, 420, L18

Hu, E. M., et al. 2002, ApJL, 568, L75

Irwin, J., \& Shmakova, M. 2006, ApJ, 645, 17

Ishigaki, M., et al. 2015, ApJ, 799, 12

Jauzac, M., et al. 2015, MNRAS, 452, 1437

Jee, M. J., et al. 2007, ApJ, 661, 728

Jha, S., Riess, A. G., \& Kirshner, R. P. 2007, ApJ, 659, 122

Johnson, T. L., et al. 2014, ApJ, 797, 48

Jones, D. O., et al. 2013, ApJ, 768, 166

Jones, T., et al. 2010, ApJL, 725, L176

Jullo, E., \& Kneib, J.-P. 2009, MNRAS, 395, 1319

Jullo, E., et al. 2007, NJPh, 9, 447

Kassiola, A., \& Kovner, I. 1993, ApJ, 417, 450

Kelly, P. L., et al. 2010, ApJ, 715, 743

Kelly, P. L., et al. 2015, Sci, 347, 1123

Kessler, R., et al. 2009a, ApJS, 185, 32

Kessler, R., et al. 2009b, PASP, 121, 1028

Kessler, R., et al. 2013, ApJ, 764, 48

Kneib, J.-P., et al. 1996, ApJ, 471, 643

Kneib, J.-P., et al. 2004, ApJ, 607, 697

Kneib, J.-P., et al. 2011, ascl soft

Kochanek, C. S. 1991, ApJ, 373, 354

Kochanek, C. S. 2006, in Saas-Fee Advanced Course 33: Gravitational

Lensing: Strong, Weak and Micro, ed. G. Meylan et al.

Kolatt, T. S., \& Bartelmann, M. 1998, MNRAS, 296, 763

Kovner, I., \& Paczynski, B. 1988, ApJL, 335, L9

Lam, D., et al. 2014, ApJ, 797, 98

Landsman, W. B. 1993, in ASP Conf. Ser. 52, Astronomical Data Analysis Software and Systems II, ed. R. J. Hanisch, R. J. V. Brissenden \& J. Barnes (San Francisco, CA: ASP), 246

Li, W., et al. 2011a, Natur, 480, 348

Li, W., et al. 2011b, MNRAS, 412, 1441

Li, X., Hjorth, J., \& Richard, J. 2012, JCAP, 11, 15

Liddle, A. R. 2009, ARNPS, 59, 95

Liesenborgs, J., \& de Rijcke, S. 2012, MNRAS, 425

Liesenborgs, J., De Rijcke, S., \& Dejonghe, H. 2006, MNRAS, 367, 1209

Liesenborgs, J., et al. 2007, MNRAS, 380, 1729

Linder, E. V. 2011, PhRvD, 84, 123529

Liu, Y., \& Modjaz, M. 2014, arXiv:1405.1437

Livermore, R. C., et al. 2015, arXiv:1503.07873

Maizy, A., et al. 2010, A\&A, 509, A105

Maoz, D. 1995, ApJL, 455, L115

Marriner, J., et al. 2011, ApJ, 740, 72

Mazzali, P. A., et al. 2014, MNRAS, 439, 1959

McCully, C., et al. 2014, MNRAS, 443, 3631

Ménard, B., Kilbinger, M., \& Scranton, R. 2010a, MNRAS, 406, 1815

Ménard, B., et al. 2010b, MNRAS, 405, 1025

Meneghetti, M., et al. 2010, A\&A, 514, A93

Meneghetti, M., et al. 2014, ApJ, 797, 34

Merten, J., et al. 2009, A\&A, 500, 681

Merten, J., et al. 2011, MNRAS, 417, 333
Mohammed, I., et al. 2014, MNRAS, 439, 2651

Mosher, J., et al. 2014, ApJ, 793, 16

Navarro, J. F., Frenk, C. S., \& White, S. D. M. 1997, ApJ, 490, 493

Nordin, J., et al. 2014, MNRAS, 440, 2742

Nugent, P. E., et al. 2011, Natur, 480, 344

Oguri, M. 2010, PASJ, 62, 1017

Oguri, M. 2015, MNRAS, 449, L86

Oguri, M., \& Kawano, Y. 2003, MNRAS, 338, L25

Okabe, N., Okura, Y., \& Futamase, T. 2010a, ApJ, 713, 291

Okabe, N., \& Umetsu, K. 2008, PASJ, 60, 345

Okabe, N., et al. 2010b, PASJ, 62, 811

Patel, B., et al. 2014, ApJ, 786, 9

Peng, C. Y., et al. 2002, AJ, 124, 266

Planck Collaboration et al. 2015, arXiv:1502.01589

Ponente, P. P., \& Diego, J. M. 2011, A\&A, 535, A119

Postman, M., et al. 2012, ApJS, 199, 25

Press, W. H. 1997, in Unsolved Problems in Astrophysics, ed. J. N. Bahcall \& J. P. Ostriker, 49

Refsdal, S. 1964, MNRAS, 128, 307

Rest, A., et al. 2014, ApJ, 795, 44

Richard, J., et al. 2006, A\&A, 456, 861

Richard, J., et al. 2008, ApJ, 685, 705

Richard, J., et al. 2014, MNRAS, 444, 268

Riehm, T., et al. 2011, A\&A, 536, A94

Riess, A. G., et al. 2001, ApJ, 560, 49

Riess, A. G., et al. 2007, ApJ, 659, 98

Rigault, M., et al. 2013, A\&A, 560, A66

Rodney, S. A., et al. 2012, ApJ, 746, 5

Rodney, S. A., et al. 2014, AJ, 148, 13

Rodney, S. A., et al. 2015, AJ, in press (arXiv:1508.03100)

Rubin, D., et al. 2013, ApJ, 763, 35

Saini, T. D., Raychaudhury, S., \& Shchekinov, Y. A. 2000, A\&A, 363, 349

Schenker, M. A., et al. 2012, ApJ, 744, 179

Schmidt, K. B., et al. 2014, ApJL, 782, L36

Schneider, P., \& Seitz, C. 1995, A\&A, 294, 411

Schneider, P., \& Sluse, D. 2014, A\&A, 564, A103

Scolnic, D., et al. 2014a, ApJ, 795, 45

Scolnic, D. M., et al. 2014b, ApJ, 780, 37

Seitz, C., \& Schneider, P. 1997, A\&A, 318, 687

Sendra, I., et al. 2014, MNRAS, 437, 2642

Shafer, D. L., \& Huterer, D. 2014, PhRvD, 89, 063510

Sharon, K., \& Johnson, T. L. 2015, ApJ, 800, 26

Smartt, S. J., et al. 2009, MNRAS, 395, 1409

Sonnenfeld, A., Bertin, G., \& Lombardi, M. 2011, A\&A, 532, A37

Stark, D. P., et al. 2008, Natur, 455, 775

Stetson, P. B. 1987, PASP, 99, 191

Stickel, M., et al. 2002, A\&A, 383, 367

Strolger, L.-G., et al. 2004, ApJ, 613, 200

Sullivan, M., et al. 2000, MNRAS, 319, 549

Sullivan, M., et al. 2010, MNRAS, 406, 782

Sullivan, M., et al. 2011, ApJ, 737, 102

Suzuki, N., et al. 2012, ApJ, 746, 85

Taylor, E. N., et al. 2011, MNRAS, 418, 1587

Tonry, J., \& Davis, M. 1979, AJ, 84, 1511

Treu, T., et al. 2015, submitted

Wang, X., et al. 2012, ApJ, 749, 126

Wang, X., et al. 2015, arXiv:1504.02405

Wong, K. C., et al. 2011, ApJ, 726, 84

Wuyts, E., et al. 2014, ApJ, 781, 61

Yuan, T.-T., et al. 2011, ApJL, 732, L14

Zheng, W., et al. 2012, Natur, 489, 406

Zitrin, A., et al. 2009, MNRAS, 396, 1985

Zitrin, A., et al. 2013, ApJL, 762, L30

Zitrin, A., et al. 2014, ApJL, 793, L12

Zitrin, A., et al. 2015, ApJ, 801, 44 